\title{
Parameter dependence of solutions of the Cauchy-Riemann equation on weighted spaces of smooth functions
}

\section{Karsten Kruse ${ }^{1}$ (D)}

Received: 31 December 2019 / Accepted: 26 April 2020 / Published online: 30 May 2020

(c) The Author(s) 2020

\begin{abstract}
Let $\Omega$ be an open subset of $\mathbb{R}^{2}$ and $E$ a complete complex locally convex Hausdorff space. The purpose of this paper is to find conditions on certain weighted Fréchet spaces $\mathcal{E} \mathcal{V}(\Omega)$ of smooth functions and on the space $E$ to ensure that the vector-valued Cauchy-Riemann operator $\bar{\partial}: \mathcal{E} \mathcal{V}(\Omega, E) \rightarrow \mathcal{E} \mathcal{V}(\Omega, E)$ is surjective. This is done via splitting theory and positive results can be interpreted as parameter dependence of solutions of the CauchyRiemann operator.
\end{abstract}

Keywords Cauchy-Riemann · Parameter dependence · Weight · Smooth · Solvability · Vector-valued

Mathematics Subject Classification 35A01 - 35B30 - 32W05 · 46A63 - 46A32 - 46E40

\section{Introduction}

Let $E$ be a linear space of functions on a set $U$ and $P(\partial): \mathcal{F}(\Omega) \rightarrow \mathcal{F}(\Omega)$ be a linear partial differential operator with constant coefficients which acts continuously on a locally convex Hausdorff space of (generalized) differentiable scalar-valued functions $\mathcal{F}(\Omega)$ on an open set $\Omega \subset \mathbb{R}^{n}$. We call the elements of $U$ parameters and say that a family $\left(f_{\lambda}\right)_{\lambda \in U}$ in $\mathcal{F}(\Omega)$ depends on a parameter w.r.t. $E$ if the map $\lambda \mapsto f_{\lambda}(x)$ is an element of $E$ for every $x \in \Omega$. The question of parameter dependence is whether for every family $\left(f_{\lambda}\right)_{\lambda \in U}$ in $\mathcal{F}(\Omega)$ depending on a parameter w.r.t. $E$ there is a family $\left(u_{\lambda}\right)_{\lambda \in U}$ in $\mathcal{F}(\Omega)$ with the same kind of parameter dependence which solves the partial differential equation

$$
P(\partial) u_{\lambda}=f_{\lambda}, \quad \lambda \in U .
$$

In particular, it is the question of $\mathcal{C}^{k}$-smooth (holomorphic, distributional, etc.) parameter dependence if $E$ is the space $\mathcal{C}^{k}(U)$ of $k$-times continuously partially differentiable functions on an open set $U \subset \mathbb{R}^{d}$ (the space $\mathcal{O}(U)$ of holomorphic functions on an open set $U \subset \mathbb{C}$, the space of distributions $\mathcal{D}(V)^{\prime}$ on an open set $V \subset \mathbb{R}^{d}$ where $U=\mathcal{D}(V)$, etc.).

$\bowtie$ Karsten Kruse

karsten.kruse@tuhh.de

1 Institute of Mathematics, Hamburg University of Technology, 21073 Hamburg, Germany 
The question of parameter dependence has been subject of extensive research varying in the choice of the spaces $E, \mathcal{F}(\Omega)$ and the properties of the partial differential operator $P(\partial)$, e.g. being (hypo)elliptic, parabolic or hyperbolic. Even partial differential differential operators $P_{\lambda}(\partial)$ where the coefficients also depend $\mathcal{C}^{k}([0,1])$-smoothly [49], $\mathcal{C}^{\infty}$-smoothly [61], holomorphically [50,61] or differentiable resp. real analytic [13] on the parameter $\lambda$ were considered. The case that the coefficients of the partial differential differential operator $P(x, \partial)$ are non-constant functions in $x \in \Omega$ was treated for $\mathcal{F}(\Omega)=\mathscr{A}\left(\mathbb{R}^{n}\right)$, the space of real analytic functions on $\mathbb{R}^{n}$, as well [3].

The answer to the question of $\mathcal{C}^{k}$-smooth (holomorphic, distributional, etc.) parameter dependence is obviously affirmative if $P(\partial)$ has a linear continuous right inverse. The problem to determine those $P(\partial)$ which have such a right inverse was posed by Schwartz in the early 1950s (see [21, p. 680]). In the case that $\mathcal{F}(\Omega)$ is the space of $\mathcal{C}^{\infty}$-smooth functions or distributions on an open set $\Omega \subset \mathbb{R}^{n}$ the problem was solved in [51,52] and in the case of ultradifferentiable functions or ultradistributions in [53] by means of Phragmén-Lindelöf type conditions. The case that $\mathcal{F}(\Omega)$ is a weighted space of $\mathcal{C}^{\infty}$-smooth functions on $\Omega=\mathbb{R}^{n}$ or its dual was handled in [40], even for some $P(x, \partial)$ with smooth coefficients, the case of tempered distributions in [38] and of Fourier (ultra-)hyperfunctions in [44,45]. For Hörmander's spaces $B_{p, K}^{l o c}(\Omega)$ as $\mathcal{F}(\Omega)$ the problem was studied in [25].

The necessary condition of surjectivity of the partial differential operator $P(\partial)$ was studied in many papers, e.g. in $[1,23,28,48,67]$ on $\mathcal{C}^{\infty}$-smooth functions and distributions, in $[9,26,43]$ on real analytic functions, in $[8,14]$ on Gevrey classes, in $[10,12,41,42,55]$ on ultradifferentiable functions of Roumieu type, in [22] on ultradistributions of Beurling type, in $[7,11]$ on ultradifferentiable functions and ultradistributions and in [47] on the multiplier space $\mathcal{O}_{M}$.

However, if $P(\partial): \mathcal{C}^{\infty}(\Omega) \rightarrow \mathcal{C}^{\infty}(\Omega), \Omega \subset \mathbb{R}^{n}$ open, is elliptic, then $P(\partial)$ has a linear right inverse (by means of a Hamel basis of $\mathcal{C}^{\infty}(\Omega)$ ) and it has a continuous right inverse due to Michael's selection theorem [56, Theorem 3.2", p. 367] and [29, Satz 9.28, p. 217], but $P(\partial)$ has no linear continuous right inverse if $n \geq 2$ by a result of Grothendieck [62, Theorem C.1, p. 109]. Nevertheless, the question of parameter dependence w.r.t. $E$ has a positive answer for several locally convex Hausdorff spaces $E$ due to tensor product techniques. In this case the question of parameter dependence obviously has a positive answer if the topology of $E$ is stronger than the topology of pointwise convergence on $U$ and

$$
P(\partial)^{E}: \mathcal{C}^{\infty}(\Omega, E) \rightarrow \mathcal{C}^{\infty}(\Omega, E)
$$

is surjective where $\mathcal{C}^{\infty}(\Omega, E)$ is the space of $\mathcal{C}^{\infty}$-smooth $E$-valued functions on $\Omega$ and $P(\partial)^{E}$ the version of $P(\partial)$ for $E$-valued functions. From Grothendieck's classical theory of tensor products [24] and the surjectivity of $P(\partial)$ it follows that $P(\partial)^{E}$ is also surjective if $E$ is a Fréchet space. In general, Grothendieck's theory of tensor products can be applied if $P(\partial)$ is surjective and $\mathcal{F}(\Omega)$ a nuclear Fréchet space. However, the surjectivity of $P(\partial)^{E}, n \geq 2$, can even be extended beyond the class of Fréchet spaces $E$ due to the splitting theory of Vogt for Fréchet spaces [64,65] and of Bonet and Domański for PLS-spaces [4,6] if, in addition, ker $P(\partial)$ has the property $(\Omega)$ and $E$ is the dual of a Fréchet space with the property $(D N)$ or an ultrabornological PLS-space with the property $(P A)$. The splitting theory of Bonet and Domański can also be applied if $\mathcal{F}(\Omega)$ is a non-Fréchet PLS-space and for PLH-spaces $\mathcal{F}(\Omega)$, e.g. $\mathcal{D}_{L^{2}}$ and $B_{2, \kappa}^{\text {loc }}(\Omega)$ which are non-PLS-spaces, the splitting theory of Dierolf and Sieg $[15,16]$ is available. For applications we refer the reader to the already mentioned papers $[4,6,15,16,64,65]$ as well as $[5,18]$ where $\mathcal{F}(\Omega)$ is the space of ultradistributions of Beurling type or of ultradifferentiable functions of Roumieu type and $E$, amongst others, the space 
of real analytic functions and to [30] where $\mathcal{F}(\Omega)$ is the space of $\mathcal{C}^{\infty}$-smooth functions or distributions.

Notably, the preceding results imply that the inhomogeneous Cauchy-Riemann equation with a right-hand side $f \in \mathcal{E}(\Omega, E):=\mathcal{C}^{\infty}(\Omega, E)$, where $\Omega \subset \mathbb{R}^{2}$ is open and $E$ a locally convex Hausdorff space over $\mathbb{C}$ whose topology is induced by a system of seminorms $\left(p_{\alpha}\right)_{\alpha \in \mathfrak{A}}$, given by

$$
\bar{\partial}^{E} u:=(1 / 2)\left(\partial_{1}^{E}+i \partial_{2}^{E}\right) u=f
$$

has a solution $u \in \mathcal{E}(\Omega, E)$ if $E$ is a Fréchet space or $E:=F_{b}^{\prime}$ where $F$ is a Fréchet space satisfying the condition $(D N)$ or if $E$ is an ultrabornological PLS-space having the property $(P A)$. Among these spaces $E$ are several spaces of distributions like $\mathcal{D}(V)^{\prime}$, the space of tempered distributions, the space of ultradistributions of Beurling type etc. In the present paper we study this problem under the constraint that the right-hand side $f$ fulfils additional growth conditions given by an increasing family of positive continuous functions $\mathcal{V}:=\left(v_{n}\right)_{n \in \mathbb{N}}$ on an increasing sequence of open subsets $\left(\Omega_{n}\right)_{n \in \mathbb{N}}$ of $\Omega$ with $\Omega=\bigcup_{n \in \mathbb{N}} \Omega_{n}$, namely,

$$
|f|_{n, m, \alpha}:=\sup _{\substack{x \in \Omega_{n} \\ \beta \in \mathbb{N}_{0}^{2},|\beta| \leq m}} p_{\alpha}\left(\left(\partial^{\beta}\right)^{E} f(x)\right) v_{n}(x)<\infty
$$

for every $n \in \mathbb{N}, m \in \mathbb{N}_{0}$ and $\alpha \in \mathfrak{A}$. Let us call the space of such functions $\mathcal{E} \mathcal{V}(\Omega, E)$. Our interest is in conditions on $\mathcal{V}$ and $\left(\Omega_{n}\right)_{n \in \mathbb{N}}$ such that there is a solution $u \in \mathcal{E} \mathcal{V}(\Omega, E)$ of (1), i.e. we search for conditions that guarantee the surjectivity of

$$
\bar{\partial}^{E}: \mathcal{E} \mathcal{V}(\Omega, E) \rightarrow \mathcal{E} \mathcal{V}(\Omega, E) .
$$

Using Grothendieck's theory of tensor products, this was already done in [33] in the case that $E$ is a Fréchet space. In the present paper we want to extend this result beyond the class of Fréchet spaces $E$. Concerning the sequence $\left(\Omega_{n}\right)_{n \in \mathbb{N}}$, we concentrate on the case that it is a sequence of strips along the real axis, i.e. $\Omega_{n}:=\{z \in \mathbb{C}|| \operatorname{Im}(z) \mid<n\}$. The case that this sequence has holes along the real axis is treated in [35].

Let us briefly outline the content of our paper. In Sect. 2 we summarise the necessary definitions and preliminaries which are needed in the subsequent sections. In Sect. 3 we recall the definitions of the topological invariants $(\Omega),(D N)$ and $(P A)$ and provide some examples of spaces $E$ having these invariants. Then we prove our main result on the surjectivity of Cauchy-Riemann operator on $\mathcal{E} \mathcal{V}(\Omega, E)$ which depends on these invariants (see Theorem $5)$. To apply our main result, the kernel ker $\bar{\partial}$ needs to have $(\Omega)$ and in Sect. 4 we provide sufficient conditions on the weights and the sequence $\left(\Omega_{n}\right)_{n \in \mathbb{N}}$ which guarantee $(\Omega)$ (see Theorem 10 and Corollary 13). We close this section with a special case of our main theorem where $\left(\Omega_{n}\right)_{n \in \mathbb{N}}$ is a sequence of strips along the real axis (see Corollary 17) and for example $v_{n}(z):=\exp \left(a_{n}|\operatorname{Re}(z)|^{\gamma}\right)$ for some $0<\gamma \leq 1$ and $a_{n} \nearrow 0$ (see Corollary 18).

\section{Notation and preliminaries}

The notation and preliminaries are essentially the same as in $[33,36$, Sect. 2]. We define the distance of two subsets $M_{0}, M_{1} \subset \mathbb{R}^{2}$ w.r.t. a norm $\|\cdot\|$ on $\mathbb{R}^{2}$ via

$$
\mathrm{d}^{\|\cdot\|}\left(M_{0}, M_{1}\right):= \begin{cases}\inf _{x \in M_{0}, y \in M_{1}}\|x-y\|, & M_{0}, M_{1} \neq \emptyset, \\ \infty, & M_{0}=\emptyset \text { or } M_{1}=\emptyset .\end{cases}
$$


Moreover, we denote by $\|\cdot\|_{\infty}$ the sup-norm, by $|\cdot|$ the Euclidean norm on $\mathbb{R}^{2}$, by $\mathbb{B}_{r}(x):=$ $\left\{w \in \mathbb{R}^{2}|| w-x \mid<r\right\}$ the Euclidean ball around $x \in \mathbb{R}^{2}$ with radius $r>0$ and identify $\mathbb{R}^{2}$ and $\mathbb{C}$ as (normed) vector spaces. We denote the complement of a subset $M \subset \mathbb{R}^{2}$ by $M^{C}:=\mathbb{R}^{2} \backslash M$, the closure of $M$ by $\bar{M}$ and the boundary of $M$ by $\partial M$. For a function $f: M \rightarrow \mathbb{C}$ and $K \subset M$ we denote by $f_{\mid K}$ the restriction of $f$ to $K$ and by

$$
\|f\|_{K}:=\sup _{x \in K}|f(x)|
$$

the sup-norm on $K$. By $L^{1}(\Omega)$ we denote the space of (equivalence classes of) $\mathbb{C}$-valued Lebesgue integrable functions on a measurable set $\Omega \subset \mathbb{R}^{2}$ and by $L^{q}(\Omega), q \in \mathbb{N}$, the space of functions $f$ such that $f^{q} \in L^{1}(\Omega)$. If $\left(a_{n}\right)_{n \in \mathbb{N}}$ is a strictly increasing real sequence, we write $a_{n} \nearrow 0$ resp. $a_{n} \nearrow \infty$ if $a_{n}<0$ for all $n \in \mathbb{N}$ and $\lim _{n \rightarrow \infty} a_{n}=0$ resp. $a_{n} \geq 0$ for all $n \in \mathbb{N}$ and $\lim _{n \rightarrow \infty} a_{n}=\infty$.

By $E$ we always denote a non-trivial locally convex Hausdorff space over the field $\mathbb{C}$ equipped with a directed fundamental system of seminorms $\left(p_{\alpha}\right)_{\alpha \in \mathfrak{A}}$. If $E=\mathbb{C}$, then we set $\left(p_{\alpha}\right)_{\alpha \in \mathfrak{A}}:=\{|\cdot|\}$. Further, we denote by $L(F, E)$ the space of continuous linear maps from a locally convex Hausdorff space $F$ to $E$ and sometimes write $\langle T, f\rangle:=T(f), f \in F$, for $T \in L(F, E)$. If $E=\mathbb{C}$, we write $F^{\prime}:=L(F, \mathbb{C})$ for the dual space of $F$. If $F$ and $E$ are (linearly topologically) isomorphic, we write $F \cong E$. We denote by $L_{t}(F, E)$ the space $L(F, E)$ equipped with the locally convex topology of uniform convergence on the finite subsets of $F$ if $t=\sigma$, on the precompact subsets of $F$ if $t=\gamma$, on the absolutely convex, compact subsets of $F$ if $t=\kappa$ and on the bounded subsets of $F$ if $t=b$.

The so-called $\varepsilon$-product of Schwartz is defined by

$$
F \varepsilon E:=L_{e}\left(F_{\kappa}^{\prime}, E\right)
$$

where $L\left(F_{\kappa}^{\prime}, E\right)$ is equipped with the topology of uniform convergence on equicontinuous subsets of $F^{\prime}$. This definition of the $\varepsilon$-product coincides with the original one by Schwartz [59, Chap. I, Sect. 1, Définition, p. 18].

We recall the following well-known definitions concerning continuous partial differentiability of vector-valued functions (c.f. [34, p. 237]). A function $f: \Omega \rightarrow E$ on an open set $\Omega \subset \mathbb{R}^{2}$ to $E$ is called continuously partially differentiable ( $f$ is $\mathcal{C}^{1}$ ) if for the $n$-th unit vector $e_{n} \in \mathbb{R}^{2}$ the limit

$$
\left(\partial^{e_{n}}\right)^{E} f(x):=\left(\partial_{n}\right)^{E} f(x):=\lim _{\substack{h \rightarrow 0 \\ h \in \mathbb{R}, h \neq 0}} \frac{f\left(x+h e_{n}\right)-f(x)}{h}
$$

exists in $E$ for every $x \in \Omega$ and $\left(\partial^{e_{n}}\right)^{E} f$ is continuous on $\Omega\left(\left(\partial^{e_{n}}\right)^{E} f\right.$ is $\left.\mathcal{C}^{0}\right)$ for every $n \in\{1,2\}$. For $k \in \mathbb{N}$ a function $f$ is said to be $k$-times continuously partially differentiable ( $f$ is $\mathcal{C}^{k}$ ) if $f$ is $\mathcal{C}^{1}$ and all its first partial derivatives are $\mathcal{C}^{k-1}$. A function $f$ is called infinitely continuously partially differentiable $\left(f\right.$ is $\mathcal{C}^{\infty}$ ) if $f$ is $\mathcal{C}^{k}$ for every $k \in \mathbb{N}$. The linear space of all functions $f: \Omega \rightarrow E$ which are $\mathcal{C}^{\infty}$ is denoted by $\mathcal{C}^{\infty}(\Omega, E)$. Let $f \in \mathcal{C}^{\infty}(\Omega, E)$. For $\beta=\left(\beta_{n}\right) \in \mathbb{N}_{0}^{2}$ we set $\left(\partial^{\beta_{n}}\right)^{E} f:=f$ if $\beta_{n}=0$, and

$$
\left(\partial^{\beta_{n}}\right)^{E} f:=\underbrace{\left(\partial^{e_{n}}\right)^{E} \cdots\left(\partial^{e_{n}}\right)^{E}}_{\beta_{n} \text {-times }} f
$$

if $\beta_{n} \neq 0$ as well as

$$
\left(\partial^{\beta}\right)^{E} f:=\left(\partial^{\beta_{1}}\right)^{E}\left(\partial^{\beta_{2}}\right)^{E} f .
$$


Due to the vector-valued version of Schwarz' theorem $\left(\partial^{\beta}\right)^{E} f$ is independent of the order of the partial derivatives on the right-hand side, we call $|\beta|:=\beta_{1}+\beta_{2}$ the order of differentiation and write $\partial^{\beta} f:=\left(\partial^{\beta}\right)^{\mathbb{C}} f$.

A function $f: \Omega \rightarrow E$ on an open set $\Omega \subset \mathbb{C}$ to $E$ is called holomorphic if the limit

$$
\left(\frac{\partial}{\partial z}\right)^{E} f\left(z_{0}\right):=\lim _{\substack{h \rightarrow 0 \\ h \in \mathbb{C}, h \neq 0}} \frac{f\left(z_{0}+h\right)-f\left(z_{0}\right)}{h}
$$

exists in $E$ for every $z_{0} \in \Omega$ and the space of such functions is denoted by $\mathcal{O}(\Omega, E)$. The exact definition of the spaces from the introduction is as follows.

Definition 1 [34, Definition 3.2, p. 238] Let $\Omega \subset \mathbb{R}^{2}$ be open and $\left(\Omega_{n}\right)_{n \in \mathbb{N}}$ a family of non-empty open sets such that $\Omega_{n} \subset \Omega_{n+1}$ and $\Omega=\bigcup_{n \in \mathbb{N}} \Omega_{n}$. Let $\mathcal{V}:=\left(v_{n}\right)_{n \in \mathbb{N}}$ be a countable family of positive continuous functions $v_{n}: \Omega \rightarrow(0, \infty)$ such that $v_{n} \leq v_{n+1}$ for all $n \in \mathbb{N}$. We call $\mathcal{V}$ a directed family of continuous weights on $\Omega$ and set for $n \in \mathbb{N}$

(a)

$$
\mathcal{E} v_{n}\left(\Omega_{n}, E\right):=\left\{\left.f \in \mathcal{C}^{\infty}\left(\Omega_{n}, E\right)\left|\forall \alpha \in \mathfrak{A}, m \in \mathbb{N}_{0}^{2}:\right| f\right|_{n, m, \alpha}<\infty\right\}
$$

and

$$
\mathcal{E} \mathcal{V}(\Omega, E):=\left\{f \in \mathcal{C}^{\infty}(\Omega, E) \mid \forall n \in \mathbb{N}: f_{\mid \Omega_{n}} \in \mathcal{E} v_{n}\left(\Omega_{n}, E\right)\right\}
$$

where

$$
|f|_{n, m, \alpha}:=\sup _{\substack{x \in \Omega_{n} \\ \beta \in \mathbb{N}_{0}^{2},|\beta| \leq m}} p_{\alpha}\left(\left(\partial^{\beta}\right)^{E} f(x)\right) v_{n}(x) .
$$

(b)

$$
\mathcal{E} v_{n, \bar{\partial}}\left(\Omega_{n}, E\right):=\left\{f \in \mathcal{E} v_{n}\left(\Omega_{n}, E\right) \mid f \in \operatorname{ker} \bar{\partial}^{E}\right\}
$$

and

$$
\mathcal{E} \mathcal{V}_{\bar{\partial}}(\Omega, E):=\left\{f \in \mathcal{E} \mathcal{V}(\Omega, E) \mid f \in \operatorname{ker} \bar{\partial}^{E}\right\}
$$

(c)

$$
\mathcal{O} v_{n}\left(\Omega_{n}, E\right):=\left\{\left.f \in \mathcal{O}\left(\Omega_{n}, E\right)|\forall \alpha \in \mathfrak{A}:| f\right|_{n, \alpha}<\infty\right\}
$$

and

$$
\mathcal{O V}(\Omega, E):=\left\{f \in \mathcal{O}(\Omega, E) \mid \forall n \in \mathbb{N}: f_{\mid \Omega_{n}} \in \mathcal{O} v_{n}\left(\Omega_{n}, E\right)\right\}
$$

where

$$
|f|_{n, \alpha}:=\sup _{x \in \Omega_{n}} p_{\alpha}(f(x)) v_{n}(x) .
$$

The subscript $\alpha$ in the notation of the seminorms is omitted in the $\mathbb{C}$-valued case. The letter $E$ is omitted in the case $E=\mathbb{C}$ as well, e.g. we write $\mathcal{E} v_{n}\left(\Omega_{n}\right):=\mathcal{E} v_{n}\left(\Omega_{n}, \mathbb{C}\right)$ and $\mathcal{E} \mathcal{V}(\Omega):=\mathcal{E} \mathcal{V}(\Omega, \mathbb{C})$. 
A projective limit $F$ of a sequence of locally convex Hausdorff spaces $\left(F_{n}\right)_{n \in \mathbb{N}}$ is called weakly reduced if for every $n \in \mathbb{N}$ there is $m \in \mathbb{N}$ such that $\pi_{n}(F)$ is dense in $F_{m}$ w.r.t. the topology of $F_{n}$ where $\pi_{n}: F \rightarrow F_{n}$ is the canonical projection. The spaces $\mathcal{F} \mathcal{V}(\Omega, E)$, $\mathcal{F}=\mathcal{E}, \mathcal{O}$, are projective limits, namely, we have

$$
\mathcal{F} \mathcal{V}(\Omega, E) \cong \lim _{n \in \mathbb{N}} \mathcal{F} v_{n}\left(\Omega_{n}, E\right)
$$

where the spectral maps are given by the restrictions

$$
\pi_{k, n}: \mathcal{F} v_{k}\left(\Omega_{k}, E\right) \rightarrow \mathcal{F} v_{n}\left(\Omega_{n}, E\right), f \mapsto f_{\mid \Omega_{n}}, k \geq n .
$$

\section{Main result}

In this section we prove our main result that the surjectivity of the vector-valued CauchyRiemann operator on $\mathcal{E} \mathcal{V}(\Omega, E)$ is inherited from the surjectivity on $\mathcal{E} \mathcal{V}(\Omega)$ if the kernel $\mathcal{E} \mathcal{V}_{\bar{\partial}}(\Omega)$ in the scalar-valued case has $(\Omega)$, and $E:=F_{b}^{\prime}$ where $F$ is a Fréchet space satisfying the condition $(D N)$ or $E$ is an ultrabornological PLS-space having the property $(P A)$. Therefore we recall the definitions of the topological invariants $(\Omega),(D N)$ and $(P A)$ and give some examples.

A Fréchet space $F$ with an increasing fundamental system of seminorms $\left(\|\mid \cdot\| \|_{k}\right)_{k \in \mathbb{N}}$ satisfies $(\Omega)$ if

$$
\forall p \in \mathbb{N} \exists q \in \mathbb{N} \forall k \in \mathbb{N} \exists n \in \mathbb{N}, C>0 \forall r>0: U_{q} \subset C r^{n} U_{k}+\frac{1}{r} U_{p}
$$

where $U_{k}:=\left\{x \in F\left|\|x \mid\|_{k} \leq 1\right\}\right.$ (see [54, Chap. 29, Definition, p. 367]).

A Fréchet space $\left(F,\left(\|\mid \cdot\|_{k}\right)_{k \in \mathbb{N}}\right)$ satisfies $(D N)$ by [54, Chap. 29, Definition, p. 359] if

$$
\exists p \in \mathbb{N} \forall k \in \mathbb{N} \exists n \in \mathbb{N}, C>0 \forall x \in F:\|x\|_{k}^{2} \leq C\|\| x\|\|_{p}\|x\|_{n} .
$$

A PLS-space is a projective limit $X=\lim _{N \in \mathbb{N}} X_{N}$, where the $X_{N}$ given by inductive limits $X_{N}=\underset{n \in \mathbb{N}}{\lim _{n}}\left(X_{N, n},\left|\|\cdot \mid\|_{N, n}\right)\right.$ are DFS-spaces (which are also called LS-spaces), and it satisfies $(P A)$ if

$$
\begin{array}{r}
\forall N \exists M \forall K \exists n \forall m \forall \eta>0 \exists k, C, r_{0}>0 \forall r>r_{0} \forall x^{\prime} \in X_{N}^{\prime}: \\
\qquad\left\|x^{\prime} \circ i_{N}^{M}\right\|_{M, m}^{*} \leq C\left(r^{\eta}\left\|x^{\prime} \circ i_{N}^{K}\right\|_{K, k}^{*}+\frac{1}{r}\left\|x^{\prime}\right\|_{N, n}^{*}\right)
\end{array}
$$

where $\||\cdot|\|^{*}$ denotes the dual norm of $\left\|\cdot|\||\right.$ and $i_{N}^{M}, i_{N}^{K}$ the linking maps (see [6, Sect. 4, Eq. (24), p. 577]).

Due to [63, 1.4 Lemma, p. 110] and [6, Proposition 4.2, p. 577] we have the following relation between the properties $(D N)$ and $(P A)$.

Remark 2 Let $F$ be a Fréchet-Schwartz space. Then $F$ satisfies $(D N)$ if and only if the DFS-space $E:=F_{b}^{\prime}$ satisfies $(P A)$.

Let us summarise some examples of ultrabornological PLS-spaces satisfying $(P A)$ and spaces of the form $E:=F_{b}^{\prime}$ where $F$ is a Fréchet space satisfying $(D N)$. The majority of them is already contained in [6], [19] and [64]. 
Example 3 (a) The following spaces are ultrabornological PLS-spaces with property $(P A)$ and also strong duals of a Fréchet space satisfying $(D N)$ :

- the strong dual of a power series space of inifinite type $\Lambda_{\infty}(\alpha)_{b}^{\prime}$,

- the strong dual of any space of holomorphic functions $\mathcal{O}(U)_{b}^{\prime}$ where $U$ is a Stein manifold with the strong Liouville property (for instance, for $U=\mathbb{C}^{d}$ ),

- the space of germs of holomorphic functions $\mathcal{O}(K)$ where $K$ is a completely pluripolar compact subset of a Stein manifold (for instance $K$ consists of one point),

- the space of tempered distributions $\mathcal{S}\left(\mathbb{R}^{d}\right)_{b}^{\prime}$ and the space of Fourier ultra-hyperfunctions $\mathcal{P}_{* *}^{\prime}$ (with the strong topology),

- the weighted distribution spaces $(K\{p M\})_{b}^{\prime}$ of Gelfand and Shilov if the weight $M$ satisfies

$$
\sup _{|y| \leq 1} M(x+y) \leq C \inf _{|y| \leq 1} M(x+y), \quad x \in \mathbb{R}^{d},
$$

- $\mathcal{D}(K)_{b}^{\prime}$ for any compact set $K \subset \mathbb{R}^{d}$ with non-empty interior,

- $\mathcal{C}^{\infty}(\bar{U})_{b}^{\prime}$ for any non-empty open bounded set $U \subset \mathbb{R}^{d}$ with $\mathcal{C}^{1}$-boundary.

(b) The following spaces are ultrabornological PLS-spaces with property $(P A)$ :

- an arbitrary Fréchet-Schwartz space,

- a PLS-type power series space $\Lambda_{r, s}(\alpha, \beta)$ whenever $s=\infty$ or $\Lambda_{r, s}(\alpha, \beta)$ is a Fréchet space,

- the spaces of distributions $\mathcal{D}(U)_{b}^{\prime}$ and ultradistributions of Beurling type $\mathcal{D}_{(\omega)}(U)_{b}^{\prime}$ for any open set $U \subset \mathbb{R}^{d}$,

- the kernel of any linear partial differential operator with constant coefficients in $\mathcal{D}(U)_{b}^{\prime}$ or in $\mathcal{D}_{(\omega)}(U)_{b}^{\prime}$ when $U \subset \mathbb{R}^{d}$ is open and convex,

- the space $L_{b}(X, Y)$ where $X$ has $(D N), Y$ has $(\Omega)$ and both are nuclear Fréchet spaces. In particular, $L_{b}\left(\Lambda_{\infty}(\alpha), \Lambda_{\infty}(\beta)\right)$ if both spaces are nuclear.

(c) The following spaces are strong duals of a Fréchet space satisfying $(D N)$ :

- the strong dual $F_{b}^{\prime}$ of any Banach space $F$,

- the strong dual $\lambda^{2}(A)_{b}^{\prime}$ of the Köthe space $\lambda^{2}(A)$ with a Köthe matrix $A=\left(a_{j, k}\right)_{j, k \in \mathbb{N}_{0}}$ satisfying

$$
\exists p \in \mathbb{N}_{0} \forall k \in \mathbb{N}_{0} \exists n \in \mathbb{N}_{0}, C>0: a_{j, k}^{2} \leq C a_{j, p} a_{j, n} .
$$

Proof The statement for the spaces in (a) and (b) follows from [19, Corollary 4.8, p. 1116], [54, Proposition 31.12, p. 401], [54, Proposition 31.16, p. 402] and Remark 2. The first part of statement (c) is obvious since Banach spaces clearly satisfy the property $(D N)$. The second part on the Köthe space $\lambda^{2}(A)$ follows from [29, Satz 12.11 a), p. 305].

Since we will use the $\varepsilon$-product $\mathcal{E} \mathcal{V}(\Omega) \varepsilon E$ to pass the surjectivity from $\bar{\partial}$ to $\bar{\partial}^{E}$, we remark the following which is not hard to prove (see [31, Sect. 39]).

Proposition 4 (a) Let $X$ be a semi-reflexive locally convex Hausdorff space and $Y$ a Fréchet space. Then $L_{b}\left(X_{b}^{\prime}, Y_{b}^{\prime}\right) \cong L_{b}\left(Y,\left(X_{b}^{\prime}\right)_{b}^{\prime}\right)$ via taking adjoints.

(b) Let $X$ be a Montel space and $E$ a locally convex Hausdorff space. Then $L_{b}\left(X_{b}^{\prime}, E\right) \cong$ $X \varepsilon E$ where the topological isomorphism is the identity map.

Theorem 5 Let $\mathcal{E} \mathcal{V}(\Omega)$ be a Schwartz space and $\mathcal{E} \mathcal{V}_{\bar{\partial}}(\Omega)$ a nuclear subspace satisfying property $(\Omega)$. Assume that the scalar-valued operator $\bar{\partial}: \mathcal{E} \mathcal{V}(\Omega) \rightarrow \mathcal{E} \mathcal{V}(\Omega)$ is surjective. Moreover, if 
(a) $E:=F_{b}^{\prime}$ where $F$ is a Fréchet space over $\mathbb{C}$ satisfying $(D N)$, or

(b) E is an ultrabornological PLS-space over $\mathbb{C}$ satisfying $(P A)$,

then

$$
\bar{\partial}^{E}: \mathcal{E} \mathcal{V}(\Omega, E) \rightarrow \mathcal{E} \mathcal{V}(\Omega, E)
$$

is surjective.

Proof Throughout this proof we use the notation $X^{\prime \prime}:=\left(X_{b}^{\prime}\right)_{b}^{\prime}$ for a locally convex Hausdorff space $X$. In both cases, $(a)$ and $(b)$, the space $E$ is a complete locally convex Hausdorff space. The space $\mathcal{E} \mathcal{V}(\Omega)$ is a Fréchet space by [34, Proposition 3.7, p. 240] and so its closed subspace $\mathcal{E} \mathcal{V}_{\bar{\partial}}(\Omega)$ as well. Further, $\mathcal{E} \mathcal{V}(\Omega)$ is a Schwartz space and $\mathcal{E} \mathcal{V}_{\bar{\partial}}(\Omega)$ nuclear, thus both spaces are reflexive. As the Fréchet-Schwartz space $\mathcal{E} \mathcal{V}(\Omega)$ is a Montel space,

$$
S: \mathcal{E} \mathcal{V}(\Omega) \varepsilon E \rightarrow \mathcal{E} \mathcal{V}(\Omega, E), u \longmapsto\left[z \mapsto u\left(\delta_{z}\right)\right],
$$

is a topological isomorphism by [36, 3.21 Example b), p. 14] where $\delta_{z}$ is the point-evaluation at $z \in \Omega$. We denote by $\mathcal{J}: E \rightarrow E^{\prime *}$ the canonical injection in the algebraic dual $E^{\prime *}$ of the topological dual $E^{\prime}$ and for $f \in \mathcal{E} \mathcal{V}(\Omega, E)$ we set

$$
R_{f}^{t}: \mathcal{E} \mathcal{V}(\Omega)^{\prime} \rightarrow E^{\prime \star}, y \longmapsto\left[e^{\prime} \mapsto y\left(e^{\prime} \circ f\right)\right]
$$

Then the map $f \mapsto \mathcal{J}^{-1} \circ R_{f}^{t}$ is the inverse of $S$ by [36, 3.17 Theorem, p. 12]. The sequence

$$
0 \rightarrow \mathcal{E} \mathcal{V}_{\bar{\partial}}(\Omega) \stackrel{i}{\rightarrow} \mathcal{E} \mathcal{V}(\Omega) \stackrel{\bar{\partial}}{\rightarrow} \mathcal{E} \mathcal{V}(\Omega) \rightarrow 0
$$

where $i$ means the inclusion, is a topologically exact sequence of Fréchet spaces because $\bar{\partial}$ is surjective by assumption. Let us denote by $J_{0}: \mathcal{E} \mathcal{V}_{\bar{\partial}}(\Omega) \rightarrow \mathcal{E} \mathcal{V}_{\bar{\partial}}(\Omega)^{\prime \prime}$ and $J_{1}: \mathcal{E} \mathcal{V}(\Omega) \rightarrow$ $\mathcal{E} \mathcal{V}(\Omega)^{\prime \prime}$ the canonical embeddings which are topological isomorphisms since $\mathcal{E} \mathcal{V}_{\bar{\partial}}(\Omega)$ and $\mathcal{E} \mathcal{V}(\Omega)$ are reflexive. Then the exactness of (4) implies that

$$
0 \rightarrow \mathcal{E} \mathcal{V}_{\bar{\partial}}(\Omega)^{\prime \prime} \stackrel{i_{0}}{\rightarrow} \mathcal{E} \mathcal{V}(\Omega)^{\prime \prime} \stackrel{\bar{\partial}_{1}}{\rightarrow} \mathcal{E} \mathcal{V}(\Omega)^{\prime \prime} \rightarrow 0
$$

where $i_{0}:=J_{0} \circ i \circ J_{0}^{-1}$ and $\bar{\partial}_{1}:=J_{1} \circ \bar{\partial} \circ J_{1}^{-1}$, is an exact topological sequence. Topological as the (strong) bidual of a Fréchet space is again a Fréchet space by [54, Corollary 25.10, p. 298].

(a) Let $E:=F_{b}^{\prime}$ where $F$ is a Fréchet space with $(D N)$. Then $\operatorname{Ext}^{1}\left(F, \mathcal{E} \mathcal{V}_{\bar{\partial}}(\Omega)^{\prime \prime}\right)=0$ by $\left[65,5.1\right.$ Theorem, p. 186] since $\mathcal{E} \mathcal{V}_{\bar{\partial}}(\Omega)$ satisfies $(\Omega)$ and therefore $\mathcal{E} \mathcal{V}_{\bar{\partial}}(\Omega)^{\prime \prime}$ as well. Combined with the exactness of (5) this implies that the sequence

$$
0 \rightarrow L\left(F, \mathcal{E} \mathcal{V}_{\bar{\partial}}(\Omega)^{\prime \prime}\right) \stackrel{i_{0}^{*}}{\rightarrow} L\left(F, \mathcal{E} \mathcal{V}(\Omega)^{\prime \prime}\right) \stackrel{\bar{\partial}_{1}^{*}}{\rightarrow} L\left(F, \mathcal{E} \mathcal{V}(\Omega)^{\prime \prime}\right) \rightarrow 0
$$

is exact by [57, Proposition 2.1, p. 13-14] where $i_{0}^{*}(B):=i_{0} \circ B$ and $\bar{\partial}_{1}^{*}(D):=\bar{\partial}_{1} \circ D$ for $B \in L\left(F, \mathcal{E} \mathcal{V}_{\bar{\partial}}(\Omega)^{\prime \prime}\right)$ and $D \in L\left(F, \mathcal{E} \mathcal{V}(\Omega)^{\prime \prime}\right)$. In particular, we obtain that

$$
\bar{\partial}_{1}^{*}: L\left(F, \mathcal{E} \mathcal{V}(\Omega)^{\prime \prime}\right) \rightarrow L\left(F, \mathcal{E} \mathcal{V}(\Omega)^{\prime \prime}\right)
$$

is surjective. Via $E=F_{b}^{\prime}$ and Proposition $4(X=\mathcal{E} \mathcal{V}(\Omega)$ and $Y=F)$ we have the topological isomorphism

$$
\psi:=S \circ{ }^{t}(\cdot): L\left(F, \mathcal{E} \mathcal{V}(\Omega)^{\prime \prime}\right) \rightarrow \mathcal{E} \mathcal{V}(\Omega, E), \psi(u)=\left(S \circ{ }^{t}(\cdot)\right)(u)=\left[z \mapsto{ }^{t} u\left(\delta_{z}\right)\right],
$$


and the inverse

$$
\psi^{-1}(f)=\left(S \circ{ }^{t}(\cdot)\right)^{-1}(f)=\left({ }^{t}(\cdot) \circ S^{-1}\right)(f)={ }^{t}\left(\mathcal{J}^{-1} \circ R_{f}^{t}\right), \quad f \in \mathcal{E} \mathcal{V}(\Omega, E) .
$$

Let $g \in \mathcal{E} \mathcal{V}(\Omega, E)$. Then $\psi^{-1}(g) \in L\left(F, \mathcal{E} \mathcal{V}(\Omega)^{\prime \prime}\right)$ and by the surjectivity of (6) there is $u \in L\left(F, \mathcal{E} \mathcal{V}(\Omega)^{\prime \prime}\right)$ such that $\bar{\partial}_{1}^{*} u=\psi^{-1}(g)$. So we get $\psi(u) \in \mathcal{E} \mathcal{V}(\Omega, E)$. Next, we show that $\bar{\partial}^{E} \psi(u)=g$ is valid. Let $x \in F, z \in \Omega$ and $h \in \mathbb{R}, h \neq 0$, and $e_{k}$ denote the $k$ th unit vector in $\mathbb{R}^{2}$. From

$$
\left(\frac{\delta_{z+h e_{k}}-\delta_{z}}{h}\right)(f)=\frac{f\left(z+h e_{k}\right)-f(z)}{h} \underset{h \rightarrow 0}{\rightarrow} \partial^{e_{k}} f(z),
$$

for every $f \in \mathcal{E} \mathcal{V}(\Omega)$ it follows that $\frac{\delta_{z+h e_{k}}-\delta_{z}}{h}$ converges to $\delta_{z} \circ \partial^{e_{k}}$ in $\mathcal{E} \mathcal{V}(\Omega)_{\sigma}^{\prime}$. Since the Fréchet-Schwartz space $\mathcal{E} \mathcal{V}(\Omega)$ is in particular a Montel space, we deduce that $\frac{\delta_{z+h e_{k}}-\delta_{z}}{h}$ converges to $\delta_{z} \circ \partial^{e_{k}}$ in $\mathcal{E} \mathcal{V}(\Omega)_{\gamma}^{\prime}=\mathcal{E} \mathcal{V}(\Omega)_{b}^{\prime}$ by the Banach-Steinhaus theorem. Let $B \subset F$ be bounded. As ${ }^{t} u \in L\left(\mathcal{E} \mathcal{V}(\Omega)_{b}^{\prime}, F_{b}^{\prime}\right)$, there are a bounded set $B_{0} \subset \mathcal{E} \mathcal{V}(\Omega)$ and $C>0$ such that

$$
\begin{aligned}
& \sup _{x \in B}\left|\left(\frac{{ }^{t} u\left(\delta_{z+h e_{k}}\right)-{ }^{t} u\left(\delta_{z}\right)}{h}\right)(x)-{ }^{t} u\left(\delta_{z} \circ \partial^{e_{k}}\right)(x)\right| \\
& =\sup _{x \in B}\left|{ }^{t} u\left(\frac{\delta_{z+h e_{k}}-\delta_{z}}{h}-\delta_{z} \circ \partial^{e_{k}}\right)(x)\right| \leq C \sup _{f \in B_{0}}\left|\left(\frac{\delta_{z+h e_{k}}-\delta_{z}}{h}-\delta_{z} \circ \partial^{e_{k}}\right)(f)\right| \underset{h \rightarrow 0}{\rightarrow} 0,
\end{aligned}
$$

yielding to $\left(\partial^{e_{k}}\right)^{E}(\psi(u))(z)={ }^{t} u\left(\delta_{z} \circ \partial^{e_{k}}\right)$. This implies $\bar{\partial}^{E}(\psi(u))(z)={ }^{t} u\left(\delta_{z} \circ \bar{\partial}\right)$. So for all $x \in F$ and $z \in \Omega$ we have

$$
\begin{aligned}
\bar{\partial}^{E}(\psi(u))(z)(x) & ={ }^{t} u\left(\delta_{z} \circ \bar{\partial}\right)(x)=u(x)\left(\delta_{z} \circ \bar{\partial}\right)=\left\langle\delta_{z} \circ \bar{\partial}, J_{1}^{-1}(u(x))\right\rangle \\
& =\left\langle\delta_{z}, \bar{\partial} J_{1}^{-1}(u(x))\right\rangle=\left\langle\left[J_{1} \circ \bar{\partial} \circ J_{1}^{-1}\right](u(x)), \delta_{z}\right\rangle=\left\langle\left(\bar{\partial}_{1} \circ u\right)(x), \delta_{z}\right\rangle \\
& =\left\langle\left(\bar{\partial}_{1}^{*} u\right)(x), \delta_{z}\right\rangle=\psi^{-1}(g)(x)\left(\delta_{z}\right)={ }^{t}\left(\mathcal{J}^{-1} \circ R_{g}^{t}\right)(x)\left(\delta_{z}\right) \\
& =\left(\mathcal{J}^{-1} \circ R_{g}^{t}\right)\left(\delta_{z}\right)(x)=\mathcal{J}^{-1}(\mathcal{J}(g(z))(x)=g(z)(x) .
\end{aligned}
$$

Thus $\bar{\partial}^{E}(\psi(u))(z)=g(z)$ for every $z \in \Omega$, which proves the surjectivity.

$(b)$ Let $E$ be an ultrabornological PLS-space satisfying $(P A)$. Since the nuclear Fréchet space $\mathcal{E} \mathcal{V}_{\bar{\partial}}(\Omega)$ is also a Schwartz space, its strong dual $\mathcal{E} \mathcal{V}_{\bar{\partial}}(\Omega)_{b}^{\prime}$ is a DFS-space. By [6, Theorem 4.1, p. 577] we obtain $\operatorname{Ext}_{P L S}^{1}\left(\mathcal{E} \mathcal{V}_{\bar{\partial}}(\Omega)_{b}^{\prime}, E\right)=0$ as the bidual $\mathcal{E} \mathcal{V}_{\bar{\partial}}(\Omega)^{\prime \prime}$ satisfies $(\Omega), E$ is a PLS-space satisfying $(P A)$ and condition (c) in the theorem is fulfilled because $\mathcal{E} \mathcal{V}_{\bar{\partial}}(\Omega)_{b}^{\prime}$ is the strong dual of a nuclear Fréchet space. Moreover, we have $\operatorname{Proj}^{1} E=0$ due to [66, Corollary 3.3.10, p. 46] because $E$ is an ultrabornological PLS-space. Then the exactness of the sequence (5), [6, Theorem 3.4, p. 567] and [6, Lemma 3.3, p. 567] (in the lemma the same condition (c) as in [6, Theorem 4.1, p. 577] is fulfilled and we choose $H=\mathcal{E} \mathcal{V}_{\bar{\partial}}(\Omega)^{\prime \prime}$ and $\left.F=G=\mathcal{E} \mathcal{V}(\Omega)^{\prime \prime}\right)$, imply that the sequence

$$
0 \rightarrow L\left(E_{b}^{\prime}, \mathcal{E} \mathcal{V}_{\bar{\partial}}(\Omega)^{\prime \prime}\right) \stackrel{i_{0}^{*}}{\rightarrow} L\left(E_{b}^{\prime}, \mathcal{E} \mathcal{V}(\Omega)^{\prime \prime}\right) \stackrel{\bar{\partial}_{1}^{*}}{\rightarrow} L\left(E_{b}^{\prime}, \mathcal{E} \mathcal{V}(\Omega)^{\prime \prime}\right) \rightarrow 0
$$

is exact. The maps $i_{0}^{*}$ and $\bar{\partial}_{1}^{*}$ are defined like in part $(a)$. Especially, we get that

$$
\bar{\partial}_{1}^{*}: L\left(E_{b}^{\prime}, \mathcal{E} \mathcal{V}(\Omega)^{\prime \prime}\right) \rightarrow L\left(E_{b}^{\prime}, \mathcal{E} \mathcal{V}(\Omega)^{\prime \prime}\right)
$$

is surjective. 
By [19, Remark 4.4, p. 1114] we have $L_{b}\left(\mathcal{E} \mathcal{V}(\Omega)_{b}^{\prime}, E^{\prime \prime}\right) \cong L_{b}\left(E_{b}^{\prime}, \mathcal{E} \mathcal{V}(\Omega)^{\prime \prime}\right)$ via taking adjoints since $\mathcal{E} \mathcal{V}(\Omega)$, being a Fréchet-Schwartz space, is a PLS-space and hence its strong dual an LFS-space, which is regular by [66, Corollary 6.7, 10. $\Leftrightarrow 11 .$, p. 114], and $E$ is an ultrabornological PLS-space, in particular, reflexive by [17, Theorem 3.2, p. 58]. In addition, the map

$$
T: L_{b}\left(\mathcal{E} \mathcal{V}(\Omega)_{b}^{\prime}, E^{\prime \prime}\right) \rightarrow L_{b}\left(\mathcal{E} \mathcal{V}(\Omega)_{b}^{\prime}, E\right),
$$

defined by $T(u)(y):=\mathcal{J}^{-1}(u(y))$ for $u \in L\left(\mathcal{E} \mathcal{V}(\Omega)_{b}^{\prime}, E^{\prime \prime}\right)$ and $y \in \mathcal{E} \mathcal{V}(\Omega)^{\prime}$, is a topological isomorphism because $E$ is reflexive. Due to Proposition 4 (b) we obtain the topological isomorphism

$$
\begin{array}{r}
\psi:=S \circ \mathcal{J}^{-1} \circ{ }^{t}(\cdot): L_{b}\left(E_{b}^{\prime}, \mathcal{E} \mathcal{V}(\Omega)^{\prime \prime}\right) \rightarrow \mathcal{E} \mathcal{V}(\Omega, E), \\
\psi(u)=\left[S \circ \mathcal{J}^{-1} \circ{ }^{t}(\cdot)\right](u)=\left[z \mapsto \mathcal{J}^{-1}\left({ }^{t} u\left(\delta_{z}\right)\right)\right],
\end{array}
$$

with the inverse given by

$$
\psi^{-1}(f)=\left(S \circ \mathcal{J}^{-1} \circ{ }^{t}(\cdot)\right)^{-1}(f)=\left[{ }^{t}(\cdot) \circ \mathcal{J} \circ S^{-1}\right](f)={ }^{t}\left(\mathcal{J} \circ \mathcal{J}^{-1} \circ R_{f}^{t}\right)={ }^{t}\left(R_{f}^{t}\right)
$$

for $f \in \mathcal{E} \mathcal{V}(\Omega, E)$.

Let $g \in \mathcal{E} \mathcal{V}(\Omega, E)$. Then $\psi^{-1}(g) \in L\left(E_{b}^{\prime}, \mathcal{E} \mathcal{V}(\Omega)^{\prime \prime}\right)$ and by the surjectivity of (7) there exists $u \in L\left(E_{b}^{\prime}, \mathcal{E} \mathcal{V}(\Omega)^{\prime \prime}\right)$ such that $\bar{\partial}_{1}^{*} u=\psi^{-1}(g)$. So we have $\psi(u) \in \mathcal{E} \mathcal{V}(\Omega, E)$. The last step is to show that $\bar{\partial}^{E} \psi(u)=g$. Like in part (a) we gain for every $z \in \Omega$

$$
\bar{\partial}^{E}(\psi(u))(z)=\mathcal{J}^{-1}\left({ }^{t} u\left(\delta_{z} \circ \bar{\partial}\right)\right)
$$

and for every $x \in E^{\prime}$

$$
\begin{aligned}
{ }^{t} u\left(\delta_{z} \circ \bar{\partial}\right)(x) & =u(x)\left(\delta_{z} \circ \bar{\partial}\right)=\left(\bar{\partial}_{1}^{*} u\right)(x)\left(\delta_{z}\right)=\psi^{-1}(g)(x)\left(\delta_{z}\right)={ }^{t}\left(R_{g}^{t}\right)(x)\left(\delta_{z}\right) \\
& =\delta_{z}(x \circ g)=x(g(z))=\mathcal{J}(g(z))(x) .
\end{aligned}
$$

Thus we have ${ }^{t} u\left(\delta_{z} \circ \bar{\partial}\right)=\mathcal{J}(g(z))$ and therefore $\bar{\partial}^{E}(\psi(u))(z)=g(z)$ for all $z \in \Omega$.

By Remark 2 case (a) is included in case (b) if $F$ is a Fréchet-Schwartz space. Therefore $(a)$ is only interesting for Fréchet spaces $F$ which are not Schwartz spaces. In the next more technical section we will present sufficient conditions for $\mathcal{E} \mathcal{V}_{\bar{\partial}}(\Omega)$ to have $(\Omega)$ as well as concrete examples of such spaces.

\section{$4(\Omega)$ for $\mathcal{O} \mathcal{V}$-spaces on strips and applications of the main result}

In this section we give some sufficient conditions such that the assumptions of our main result Theorem 5 are fulfilled. The outline is as follows. First, we show that $\mathcal{O} \mathcal{V}(\Omega)$ and $\mathcal{E} \mathcal{V}_{\bar{\partial}}(\Omega)$ coincide topologically under mild assumptions on the weights $\mathcal{V}$ and the sequence of sets $\left(\Omega_{n}\right)$. These mild conditions also imply that $\mathcal{E} \mathcal{V}(\Omega)$ is nuclear, in particular Schwartz, and thus its subspace $\mathcal{E} \mathcal{V}_{\bar{\partial}}(\Omega)=\mathcal{O} \mathcal{V}(\Omega)$ too. Second, we reduce the problem whether the projective limit $\mathcal{O V}(\Omega)$ has $(\Omega)$ to the problem whether it is weakly reduced in the case that the $\Omega_{n}$ are strips along the real axis and the weights have a certain structure. Third, we use a similar result for $\mathcal{E} \mathcal{V}_{\bar{\partial}}(\Omega)$ which was obtained in [33] to prove the weak reducibility of $\mathcal{O V}(\Omega)$. For corresponding results in the case that $\Omega_{n}=\Omega$ for all $n \in \mathbb{N}$ see [20, Theorem 3, p. 56], [39, 1.3 Lemma, p. 418] and [58, Theorem 1, p. 145]. We close this section with some examples of our main result. Let us start with the sufficient conditions, guaranteeing 
that the projective limit $\mathcal{E} \mathcal{V}(\Omega)$ is nuclear (if $q=1$ ). They also allow to switch from sup- to weighted $L^{q}$-seminorms which is important for the proof of surjectivity of the scalar-valued $\bar{\partial}$-operator given in [33], using Hörmander's $L^{2}$-machinery (if $q=2$ ).

Condition (PN) ([33, 3.3 Condition, p. 7]) Let $\mathcal{V}:=\left(v_{n}\right)_{n \in \mathbb{N}}$ be a directed family of continuous weights on an open set $\Omega \subset \mathbb{R}^{2}$ and $\left(\Omega_{n}\right)_{n \in \mathbb{N}}$ a family of non-empty open sets such that $\Omega_{n} \subset \Omega_{n+1}$ and $\Omega=\bigcup_{n \in \mathbb{N}} \Omega_{n}$. For every $k \in \mathbb{N}$ let there be $\rho_{k} \in \mathbb{R}$ such that $0<\rho_{k}<\mathrm{d}^{\|\cdot\|_{\infty}}\left(\{x\}, \partial \Omega_{k+1}\right)$ for all $x \in \Omega_{k}$ and let there be $q \in \mathbb{N}$ such that for any $n \in \mathbb{N}$ there is $\psi_{n} \in L^{q}\left(\Omega_{k}\right), \psi_{n}>0$, and $\mathbb{N} \ni J_{i}(n) \geq n$ and $C_{i}(n)>0$ such that for any $x \in \Omega_{k}$ :

$(P N .1) \sup _{\zeta \in \mathbb{R}^{2},\|\zeta\|_{\infty} \leq \rho_{k}} v_{n}(x+\zeta) \leq C_{1}(n) \inf _{\zeta \in \mathbb{R}^{2},\|\zeta\|_{\infty} \leq \rho_{k}} v_{J_{1}(n)}(x+\zeta)$

$(P N .2)^{q} v_{n}(x) \leq C_{2}(n) \psi_{n}(x) v_{J_{2}(n)}(x)$

Example 6 Let $\Omega:=\mathbb{R}^{2}$ and $\Omega_{n}:=\left\{x=\left(x_{i}\right) \in \mathbb{R}^{2}|| x_{2} \mid<n\right\}$. Let $0<\gamma \leq 1$ and $\left(a_{n}\right)_{n \in \mathbb{N}}$ be strictly increasing such that $a_{n} \geq 0$ for all $n \in \mathbb{N}$ or $a_{n} \leq 0$ for all $n \in \mathbb{N}$. The family $\mathcal{V}:=\left(v_{n}\right)_{n \in \mathbb{N}}$ of positive continuous functions on $\Omega$ given by

$$
v_{n}: \Omega \rightarrow(0, \infty), v_{n}(x):=e^{a_{n}\left|x_{1}\right|^{\gamma}},
$$

fulfils $v_{n} \leq v_{n+1}$ all $n \in \mathbb{N}$ and $(P N)$ for every $q \in \mathbb{N}$ with $\psi_{n}(x):=\left(1+|x|^{2}\right)^{-2}, x \in \mathbb{R}^{2}$, for every $n \in \mathbb{N}$.

The space $\mathcal{O V}(\mathbb{C})$ with this kind of weights consists of functions which are entire and exponentially growing $\left(a_{n}<0\right)$ resp. decreasing $\left(a_{n}>0\right)$ with order $\gamma$ on strips along the real axis. This example of weights and many more are included in [33, 3.7 Example, p. 9]. We restrict to this particular weights because we use it in an example for our main result.

Proposition 7 Let $\mathcal{V}:=\left(v_{n}\right)_{n \in \mathbb{N}}$ be a directed family of continuous weights on an open set $\Omega \subset \mathbb{R}^{2}$ and $\left(\Omega_{n}\right)_{n \in \mathbb{N}}$ a family of non-empty open sets such that $\Omega_{n} \subset \Omega_{n+1}$ and $\Omega=\bigcup_{n \in \mathbb{N}} \Omega_{n}$. If (PN.1) is fulfilled, then

(a) for every $n \in \mathbb{N}$ and $m \in \mathbb{N}_{0}$ there is $C>0$ such that

$$
|f|_{n, m} \leq C|f|_{2 J_{1}(n)}, \quad f \in \mathcal{O} v_{2 J_{1}(n)}\left(\Omega_{2 J_{1}(n)}\right) .
$$

(b) $\mathcal{E V}_{\bar{\partial}}(\Omega)=\mathcal{O} \mathcal{V}(\Omega)$ as Fréchet spaces.

Proof (a) Let $n \in \mathbb{N}$ and $m \in \mathbb{N}_{0}$. We note that $\Omega_{n+1} \subset \Omega_{2 J_{1}(n)}$ and $\partial^{\beta} f(x)=i^{\beta_{2}} f^{(|\beta|)}(x)$, $x \in \Omega_{2 J_{1}(n)}$, holds for all $\beta=\left(\beta_{1}, \beta_{2}\right) \in \mathbb{N}_{0}^{2}$ and $f \in \mathcal{O} \nu_{2 J_{1}(n)}\left(\Omega_{2 J_{1}(n)}\right)$ where $f^{(|\beta|)}$ is the $|\beta|$ th complex derivative of $f$. Then we obtain via (PN.1) and Cauchy's inequality

$$
\begin{aligned}
|f|_{n, m} & =\sup _{\substack{x \in \Omega_{n} \\
\beta \in \mathbb{N}_{0}^{2},|\beta| \leq m}}\left|\partial^{\beta} f(x)\right| v_{n}(x) \leq \sup _{\substack{x \in \Omega_{n} \\
\beta \in \mathbb{N}_{0}^{2},|\beta| \leq m}} \frac{|\beta| !}{\rho_{n}^{|\beta|}} \max _{\substack{\zeta \in \mathbb{R}^{2} \\
|\zeta-x|=\rho_{n}}}|f(\zeta)| v_{n}(x) \\
& \leq C_{1} \sup _{\substack{x \in \Omega_{n} \\
\beta \in \mathbb{N}_{0}^{2},|\beta| \leq m}} \frac{|\beta| !}{\rho_{n}^{|\beta|}} \max _{\substack{\zeta \in \mathbb{R}^{2} \\
|\zeta-x|=\rho_{n}}}|f(\zeta)| v_{J_{1}(n)}(\zeta) \\
& \leq C_{1} \sup _{\beta \in \mathbb{N}_{0}^{2},|\beta| \leq m} \frac{|\beta| !}{\rho_{n}^{|\beta|}} \sup _{\zeta \in \Omega_{n+1}}|f(\zeta)| v_{J_{1}(n)}(\zeta) \leq C_{1} \sup _{\beta \in \mathbb{N}_{0}^{2},|\beta| \leq m} \frac{|\beta| !}{\rho_{n}^{|\beta|}|f|_{2 J_{1}(n) .} .}
\end{aligned}
$$

(b) The space $\mathcal{E} \mathcal{V}_{\bar{\partial}}(\Omega)$ is a Fréchet space since it is a closed subspace of the Fréchet space $\mathcal{E} \mathcal{V}(\Omega)$ by [34, Proposition 3.7, p. 240]. From part (a) and $|f|_{n}=|f|_{n, 0}$ for all $n \in \mathbb{N}$ and $f \in \mathcal{E} \mathcal{V}_{\bar{\partial}}(\Omega)$ follows the statement. 
Let us come to the second part. Using special weight functions, strips along the real axis as $\Omega_{n}$ and a decomposition theorem of Langenbruch, we will see that answering the question whether $\mathcal{O V}(\Omega)$ satisfies the property $(\Omega)$ of Vogt boils down to answering whether the projective limit $\mathcal{O V}(\Omega)$ is weakly reduced. The special weights we want to consider are generated by a function $\mu$ with the following properties.

Definition 8 (strong weight generator) A continuous function $\mu: \mathbb{C} \rightarrow[0, \infty)$ is called a weight generator if $\mu(z)=\mu(|\operatorname{Re}(z)|)$ for all $z \in \mathbb{C}$, the restriction $\mu_{\mid[0, \infty)}$ is strictly increasing,

$$
\lim _{\substack{x \rightarrow \infty \\ x \in \mathbb{R}}} \frac{\ln (1+|x|)}{\mu(x)}=0
$$

and

$$
\exists \Gamma>1, C>0 \forall x \in[0, \infty): \mu(x+1) \leq \Gamma \mu(x)+C .
$$

If $\mu$ is a weight generator which fulfils the stronger condition

$$
\exists \Gamma>1 \forall n \in \mathbb{N} \exists C>0 \forall x \in[0, \infty): \mu(x+n) \leq \Gamma \mu(x)+C,
$$

then $\mu$ is called a strong weight generator.

Weight generators are introduced in [46, Definition 2.1, p. 225] and strong weight generators in [60, Definition 2.2.2, p. 43] where they are simply called weight functions resp. strong weight functions. For a weight generator $\mu$ we define the space

$$
H_{\tau}\left(S_{t}\right):=\left\{f \in \mathcal{O}\left(S_{t}\right)\left|\|f\|_{\tau, t}:=\sup _{z \in S_{t}}\right| f(z) \mid e^{\tau \mu(z)}<\infty\right\}
$$

for $t>0$ and $\tau \in \mathbb{R}$ with the strip $S_{t}:=\{z \in \mathbb{C}|| \operatorname{Im}(z) \mid<t\}$.

Theorem 9 [46, Theorem 2.2, p. 225] ${ }^{1}$ Let $\mu$ be a weight generator. There are $\tilde{t}, K_{1}, K_{2}>0$ such that for any $\tau_{0}<\tau<\tau_{2}$ there is $C_{0}=C_{0}(\operatorname{sign}(\tau))$ such that for any $0<2 t_{0}<t<$ $t_{2}<\tilde{t}$ with

$$
t_{0} \leq \min \left[K_{1}, K_{2} \sqrt{\frac{\tau-C_{0} \tau_{0}}{\tau_{2}-C_{0} \tau_{0}}}\right]
$$

there is $C_{1} \geq 1$ such that for any $r \geq 0$ and any $f \in H_{\tau}\left(S_{t}\right)$ with $\|f\|_{\tau, t} \leq 1$ the following holds: there are $f_{2} \in \mathcal{O}\left(S_{t_{2}}\right)$ and $f_{0} \in \mathcal{O}\left(S_{t_{0}}\right)$ such that $f=f_{0}+f_{2}$ on $S_{t_{0}}$ and

$$
\left\|f_{0}\right\|_{C_{0} \tau_{0}, t_{0}} \leq C_{1} e^{-G r} \text { and }\left\|f_{2}\right\|_{\tau_{2}, t_{2}} \leq e^{r}
$$

where

$$
G:=K_{1} \min \left[1, \frac{t-t_{0}}{2 \widetilde{t}}, \frac{\tau-C_{0} \tau_{0}}{\tau_{2}-C_{0} \tau_{0}}\right] .
$$

To apply this theorem, we have to know the constants involved. In the following the notation of [46] is used and it is referred to the corresponding positions resp. conditions for these constants. We have

$$
\tilde{t}:=\frac{1}{4 \ln (\Gamma)}
$$

1 A superfluous constant depending on $\operatorname{sign}\left(\tau_{0}\right)$ is omitted. 
by [46, Lemma 2.4, (2.15), p. 228] with $\Gamma$ from Definition 8 such that $\Gamma \geq e^{1 / 4}$. The choice $\Gamma \geq e^{1 / 4}$ comes from wanting $\tilde{t} \leq 1$ in [46, Lemma 2.4, p. 228]. By [46, Corollary 2.6, p. 230-231] we have

$$
C_{0}:= \begin{cases}4 \Gamma B_{3}=\frac{64 \cosh (1)}{\cos (1 / 2)} \Gamma^{2}>1, \tau<0 \\ \frac{1}{4 \Gamma B_{3}}=\frac{\cos (1 / 2)}{64 \cosh (1) \Gamma^{2}}<1 \quad, \tau \geq 0\end{cases}
$$

where $B_{3}:=\frac{16 \cosh (1)}{\cos (1 / 2)} \Gamma$ by [46, Lemma 2.4, p. 228-229]. ${ }^{2}$ To get the constants $K_{1}$ and $K_{2}$, we have to analyze the conditions for $t_{0}$ in the proof of [46, Theorem 2.2, p. 225]. By the assumptions on $\tau_{0}, \tau$ and $\tau_{2}$ and the choice of $C_{0}$ we obtain

$$
\tau_{2}-C_{0} \tau_{0}>\tau_{2}-C_{0} \tau \geq \tau_{2}-\tau>0
$$

and

$$
\tau-C_{0} \tau_{0}>\tau-C_{0} \tau=\tau\left(1-C_{0}\right)>0 .
$$

By choosing $D>0$ in the proof of [46, Theorem 2.2, (2.22), p. 232-233] as $D:=$ $\frac{\tau-C_{0} \tau_{0}}{\left(\tau_{2}-C_{0} \tau_{0}\right) 2 \Gamma_{0}}$, the estimate

$$
D=\frac{\tau-C_{0} \tau_{0}}{\left(\tau_{2}-C_{0} \tau_{0}\right) 2 \Gamma_{0}}=\min \left(\frac{1}{2 \widetilde{\Gamma}}, \frac{1}{2 \widehat{\Gamma}}\right) \frac{\tau-C_{0} \tau_{0}}{\tau_{2}-C_{0} \tau_{0}} \underset{(8),(9)}{\leq} \min \left(\frac{1}{2 \widetilde{\Gamma}}, \frac{1}{2 \widehat{\Gamma}}\right) \frac{\tau-C_{0} \tau_{0}}{\tau_{2}-C_{0} \tau}
$$

holds where $\Gamma_{0}:=\max (\widetilde{\Gamma}, \widehat{\Gamma})$ with $\widetilde{\Gamma}, \widehat{\Gamma}>1$ from the proof. With $\theta \geq \frac{t-t_{0}}{2 t}$ (p. 232) we get on p. 233, below (2.24), due to the condition $t_{0} \leq T_{0}:=\min \left(\frac{t}{2}, \frac{1}{4 a^{2} B_{1} \vec{t}}\right)$,

$$
\begin{aligned}
& \min \left(\frac{\theta}{2}, D, 1\right) \geq \min \left(\frac{1}{2}, \frac{1}{2 \Gamma_{0}}\right) \min \left(\theta, \frac{\tau-C_{0} \tau_{0}}{\tau_{2}-C_{0} \tau_{0}}, 1\right) \geq \frac{1}{2 \Gamma_{0}} \min \left(\frac{t-t_{0}}{2 \widetilde{t}}, \frac{\tau-C_{0} \tau_{0}}{\tau_{2}-C_{0} \tau_{0}}, 1\right) \\
& \geq \min \left(\frac{1}{2 \Gamma_{0}}, \frac{1}{4 a^{2} B_{1} \widetilde{t}}\right) \min \left(\frac{t-t_{0}}{2 \widetilde{t}}, \frac{\tau-C_{0} \tau_{0}}{\tau_{2}-C_{0} \tau_{0}}, 1\right) \\
& =\underbrace{\min \left(\frac{1}{2 \Gamma_{0}}, \frac{1}{2 \cosh (1) \ln (\Gamma)}\right)}_{=: K_{1}} \min \left(\frac{t-t_{0}}{2 \widetilde{t}}, \frac{\tau-C_{0} \tau_{0}}{\tau_{2}-C_{0} \tau_{0}}, 1\right)=: G
\end{aligned}
$$

where $a:=\ln (\Gamma)$ (in the middle of p. 231) and $B_{1}:=2 \cosh (1)$ by the proof of [46, Lemma 2.3, p. 226-227]. The assumptions $2 t_{0}<t$ and $t_{0} \leq K_{1}$ in Theorem 9 guarantee that the condition $t_{0} \leq T_{0}$ is satisfied. Looking at the condition $t_{0} \leq T_{1}:=\sqrt{\frac{D}{a^{2} B_{1}}}$ (p. 232), we derive

$$
T_{1}=\frac{1}{\sqrt{2 \Gamma_{0} a^{2} B_{1}}} \sqrt{\frac{\tau-C_{0} \tau_{0}}{\tau_{2}-C_{0} \tau_{0}}}=\underbrace{\frac{1}{2 \sqrt{\cosh (1) \Gamma_{0}} \ln (\Gamma)}}_{=: K_{2}} \sqrt{\frac{\tau-C_{0} \tau_{0}}{\tau_{2}-C_{0} \tau_{0}}} .
$$

For the subsequent theorem we merge and modify the proofs of [60, Satz 2.2.3, p. 44] ${ }^{3}$ ( $a_{n}=n, n \in \mathbb{N}$, and $\mu$ a strong weight generator) and [32, 5.20 Theorem, p. 84] $\left(a_{n}=-1 / n\right.$, $n \in \mathbb{N}$, and $\mu=|\operatorname{Re}(\cdot)|)$.

\footnotetext{
${ }^{2}$ An error in part b) of this lemma, p. 229, is corrected here such that the term $\cos (1 / 2)=$ $\min _{|y| \leq \tilde{t}=1 /\left(2 C_{1}\right)} \cos \left(C_{1} y\right)$ appears.

3 The proof of [60, Satz 2.2.3, p. 44] relies on [60, Satz 2.2.1, p. 43] which is an announced version (without a proof) of our result Corollary 13 on weak reducibility.
} 
Theorem 10 Let $\mu$ be a strong weight generator, $a_{n} \nearrow 0$ or $a_{n} \nearrow \infty, \mathcal{V}:=\left(\exp \left(a_{n} \mu\right)\right)_{n \in \mathbb{N}}$ and $\Omega_{n}:=S_{n}$ for all $n \in \mathbb{N}$. If $\mathcal{O} \mathcal{V}(\mathbb{C})$ is weakly reduced, then $\mathcal{O V}(\mathbb{C})$ satisfies $(\Omega)$.

Proof Since $\mathcal{O V}(\mathbb{C})$ is weakly reduced, for every $n \in \mathbb{N}$ there exists $m_{n} \in \mathbb{N}$ such that $\pi_{n}(\mathcal{O V}(\mathbb{C}))$ is dense in $\mathcal{O} v_{m_{n}}\left(\Omega_{m_{n}}\right)$ w.r.t. the topology of $\mathcal{O} v_{n}\left(\Omega_{n}\right)$ where

$$
\pi_{n}: \mathcal{O} \mathcal{V}(\mathbb{C}) \rightarrow \mathcal{O} v_{n}\left(\Omega_{n}\right), \pi_{n}(f):=f_{\mid \Omega_{n}},
$$

is the canonical projection. Let $p, k \in \mathbb{N}$. As $\left(a_{n}\right)_{n \in \mathbb{N}}$ is strictly increasing and $\lim _{n \rightarrow \infty} a_{n}=$ 0 or $\lim _{n \rightarrow \infty} a_{n}=\infty$, we may choose $q \in \mathbb{N}$ such that $a_{m_{p}} / C_{0}<a_{q}$ and $2 m_{p}<q$. To use the decomposition from Theorem 9, we need a linear transformation between strips to get the decomposition on the desired strip $S_{m_{p}}$. We choose $\Gamma \geq e^{1 / 4}$ and $T \in \mathbb{R}$ such that

$$
0<T<\frac{1}{4 \max \left(q+1, m_{k}\right) \ln (\Gamma)}
$$

which also fulfils

$$
T \leq \frac{1}{m_{p}} \min \left(\frac{1}{2 \Gamma_{0}}, \frac{1}{2 \cosh (1) \ln (\Gamma)}, \frac{1}{2 \sqrt{\cosh (1) \Gamma_{0}} \ln (\Gamma)} \sqrt{\frac{a_{q}-a_{m_{p}}}{\max \left(a_{q+1}, a_{m_{k}}\right)-a_{m_{p}}}}\right) .
$$

Let

$$
\begin{aligned}
& \tau_{0}:=\frac{a_{m_{p}}}{C_{0}}, \quad \tau:=a_{q}, \quad \tau_{2}:=\max \left(a_{q+1}, a_{m_{k}}\right), \\
& t_{0}:=m_{p} T, \quad t:=q T, \quad t_{2}:=\max \left(q+1, m_{k}\right) T .
\end{aligned}
$$

By the choice of $q$ we have

$$
\tau_{0}=\frac{a_{m_{p}}}{C_{0}}<a_{q}=\tau<\max \left(a_{q+1}, a_{m_{k}}\right)=\tau_{2} .
$$

By the choice of $q$ and (10) we get

$$
0<2 t_{0}=2 m_{p} T<q T=t<\max \left(q+1, m_{k}\right) T=t_{2}<\frac{1}{4 \ln (\Gamma)}=\tilde{t} .
$$

Further, we deduce from (11) that

$$
t_{0}=m_{p} T \leq \min \left[K_{1}, K_{2} \sqrt{\frac{\tau-C_{0} \tau_{0}}{\tau_{2}-C_{0} \tau_{0}}}\right]
$$

Let $r \geq 0$ and $f \in \mathcal{O V}(\mathbb{C})$ such that $|f|_{q}=\|f\|_{a_{q}, q} \leq 1$. We set $\tilde{f}: S_{q T} \rightarrow \mathbb{C}, \tilde{f}(z):=$ $f(z / T)$, and define

$$
H_{\tau}^{\sim}\left(S_{t}\right):=\left\{g \in \mathcal{O}\left(S_{t}\right)\left|\|g\|_{\tau, t}^{\sim}:=\sup _{z \in S_{t}}\right| g(z) \mid e^{\tau \tilde{\mu}(z)}<\infty\right\}
$$

where $\tilde{\mu}:=\mu(\cdot / T)$. We note that for $\tilde{n}:=\lceil 1 / T\rceil$, where $\lceil\cdot\rceil$ is the ceiling function, there is $C>0$ such that for all $x \geq 0$

$$
\tilde{\mu}(x+1)=\mu\left(\frac{x+1}{T}\right) \leq \mu\left(\frac{x}{T}+\left\lceil\frac{1}{T}\right\rceil\right)=\mu\left(\frac{x}{T}+\tilde{n}\right) \leq \Gamma \mu\left(\frac{x}{T}\right)+C=\Gamma \tilde{\mu}(x)+C
$$


because $\mu$ is a strong weight generator. We conclude that $\tilde{\mu}$ is also a weight generator with the same $\Gamma$ as $\mu$ which is independent of $T$. Moreover, from

$$
\|\tilde{f}\|_{\tau, t}^{\sim}=\sup _{z \in S_{q T}}|\tilde{f}(z)| e^{a_{q} \tilde{\mu}(z)}=\sup _{z \in S_{q}}|f(z)| e^{a_{q} \mu(z)}=|f|_{q} \leq 1
$$

it follows by Theorem 9 that there are $\tilde{f}_{j} \in \mathcal{O}\left(S_{t_{j}}\right), j \in\{0,2\}$, such that

$$
\tilde{f}(z)=\tilde{f}_{0}(z)+\tilde{f}_{2}(z), \quad z \in S_{t_{0}}
$$

and

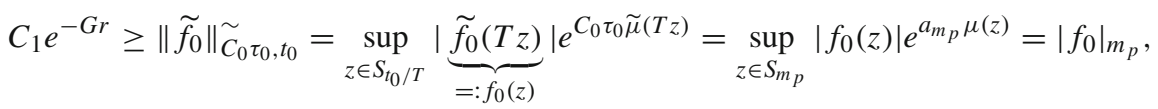

where $f_{0} \in \mathcal{O}\left(S_{m_{p}}\right)$, as well as

$$
e^{r} \geq\left\|\widetilde{f}_{2}\right\|_{\tau_{2}, t_{2}}^{\sim}=\sup _{z \in S_{t_{2} / T}}|\underbrace{\tilde{f}_{2}(T z)}_{=: f_{2}(z)}| e^{\tau_{2} \tilde{\mu}(T z)} \geq \sup _{z \in S_{m_{k}}}\left|f_{2}(z)\right| e^{a_{m_{k}} \mu(z)}=\left|f_{2}\right|_{m_{k}}
$$

where $f_{2} \in \mathcal{O}\left(S_{t_{2} / T}\right) \subset \mathcal{O}\left(S_{m_{k}}\right)$ and the inclusion is justified by the identity theorem. Furthermore, for $z \in S_{t_{0} / T}=S_{m_{p}}$ the equation

$$
f(z)=\tilde{f}(T z) \underset{(12)}{=} \widetilde{f_{0}}(T z)+\widetilde{f_{2}}(T z)=f_{0}(z)+f_{2}(z)
$$

holds, thus $f=f_{0}+f_{2}$ on $S_{m_{p}}$. By virtue of the weak reducibility of $\mathcal{O V}(\mathbb{C})$ and the choice of $m_{p}, m_{k}$ the following is valid:

$$
\forall \varepsilon>0 \exists \widehat{f_{0}}, \widehat{f_{2}} \in \mathcal{O} \mathcal{V}(\mathbb{C}): \text { (i) }\left|\widehat{f_{0}}-f_{0}\right|_{p}<\varepsilon \text { and } \text { (ii) }\left|\widehat{f_{2}}-f_{2}\right|_{k}<\varepsilon .
$$

Now, we have to consider two cases. Let $\varepsilon:=C_{1} e^{-G r}$. For $k \leq p$ we get via (15) $(i)$ that $f=\widehat{f_{0}}+\left(f_{2}+f_{0}-\widehat{f_{0}}\right)$ on $S_{m_{p}}$ so

$$
f_{2}+f_{0}-\widehat{f_{0}}=f-\widehat{f_{0}}=: \bar{f}_{2} \text { on } S_{m_{p}}
$$

where the function $\bar{f}_{2} \in \mathcal{O} \mathcal{V}(\mathbb{C})$ and thus is a holomorphic extension of the left-hand side on $\mathbb{C}$. Hence we clearly have $f=\widehat{f}_{0}+\bar{f}_{2}$ and

$$
\left|\widehat{f_{0}}\right|_{p} \leq\left|\widehat{f_{0}}-f_{0}\right|_{p}+\left|f_{0}\right|_{p} \underset{(15)(i)}{\leq} \varepsilon+\left|f_{0}\right|_{p} \leq \varepsilon+\left|f_{0}\right|_{m_{p}} \underset{(13)}{\leq} 2 C_{1} e^{-G r}=: C_{2} e^{-G r}
$$

as well as

$$
\begin{aligned}
\left|\bar{f}_{2}\right|_{k} & \leq\left|\bar{f}_{2}-f_{2}\right|_{k}+\left|f_{2}\right|_{k} \underset{(16), k \leq p}{\leq}\left|f_{0}-\widehat{f_{0}}\right|_{p}+\left|f_{2}\right|_{m_{k}} \underset{(15)(i)}{\leq} \varepsilon+\left|f_{2}\right|_{m_{k}} \\
& \leq C_{1} e^{-G r}+e^{r} \leq\left(C_{1}+1\right) e^{r}=: C_{3} e^{r} .
\end{aligned}
$$

Analogously, for $k>p$ we obtain via (15) $(i i)$ that $f=\widehat{f_{2}}+\left(f_{0}+f_{2}-\widehat{f_{2}}\right)$ on $S_{m_{p}}$ so

$$
f_{0}+f_{2}-\widehat{f_{2}}=f-\widehat{f_{2}}=: \bar{f}_{0} \text { on } S_{m_{p}}
$$


where the function $\bar{f}_{0} \in \mathcal{O} \mathcal{V}(\mathbb{C})$ and thus is a holomorphic extension of the left-hand side on $\mathbb{C}$. Hence we clearly have $f=\bar{f}_{0}+\widehat{f}_{2}$ and

$$
\begin{aligned}
\left|\bar{f}_{0}\right|_{p} & =\left|f-\widehat{f_{2}}\right|_{p} \underset{(19)}{=}\left|f_{0}+f_{2}-\widehat{f_{2}}\right|_{p} \leq\left|f_{2}-\widehat{f_{2}}\right|_{p}+\left|f_{0}\right|_{p} \underset{k>p}{\leq}\left|f_{2}-\widehat{f_{2}}\right|_{k}+\left|f_{0}\right|_{m_{p}} \\
& \leq \varepsilon+\left|f_{0}\right|_{m_{p}} \underset{(15)(i \mathrm{ii})}{\leq} 2 C_{1} e^{-G r}=C_{2} e^{-G r}
\end{aligned}
$$

as well as

$$
\left|\widehat{f_{2}}\right|_{k} \leq\left|\widehat{f_{2}}-f_{2}\right|_{k}+\left|f_{2}\right|_{k} \underset{(15)(i i)}{\leq} \varepsilon+\left|f_{2}\right|_{m_{k}} \underset{(14)}{\leq} C_{1} e^{-G r}+e^{r} \leq C_{3} e^{r} .
$$

Next, we set $n:=\lceil 1 / G\rceil$ and $C:=C_{3} e^{\ln \left(C_{2}\right) / G}$. Let $\widetilde{r}>0$. For $\widetilde{r} \geq 1$ there is $r \geq 0$ such that

$$
\tilde{r}=e^{G r-\ln \left(C_{2}\right)}=\frac{e^{G r}}{C_{2}}
$$

and we have by (17) and (18) for $k \leq p$

$$
\left|\widehat{f}_{0}\right|_{p} \leq C_{2} e^{-G r}=\frac{1}{\widetilde{r}}, \quad\left|\bar{f}_{2}\right|_{k} \leq C_{3} e^{r}=C_{3} e^{\frac{1}{G} \ln \left(C_{2}\right)} e^{\frac{1}{G}\left(G r-\ln \left(C_{2}\right)\right)}=C \widetilde{r}^{\frac{1}{G}} \underset{\widetilde{r} \geq 1}{\leq} C \widetilde{r}^{n},
$$

as well as by (20) and (21) for $k>p$

$$
\left|\bar{f}_{0}\right|_{p} \leq \frac{1}{\widetilde{r}}, \quad\left|\widehat{f}_{2}\right|_{k} \leq C \widetilde{r}^{n}
$$

For $0<\widetilde{r}<1$ we have, since $q \geq p$,

$$
|f|_{p} \leq|f|_{q} \leq 1<\frac{1}{\widetilde{r}}
$$

Thus our statement is proved.

Let us remark that the choice of the sequence $\left(a_{n}\right)_{n \in \mathbb{N}}$ in the preceding theorem does not really matter.

Remark 11 Let $\mu: \mathbb{C} \rightarrow[0, \infty)$ be continuous, $a_{n} \nearrow 0$ or $a_{n} \nearrow \infty, \mathcal{V}:=\left(\exp \left(a_{n} \mu\right)\right)_{n \in \mathbb{N}}$ and $\Omega_{n}:=S_{n}$ for all $n \in \mathbb{N}$. Set $\mathcal{V}_{-}:=(\exp ((-1 / n) \mu))_{n \in \mathbb{N}}$ and $\mathcal{V}_{+}:=(\exp (n \mu))_{n \in \mathbb{N}}$. Then

$$
\mathcal{O} \mathcal{V}(\mathbb{C}) \cong \mathcal{O} \mathcal{V}_{-}(\mathbb{C}), \quad \text { if } a_{n} \nearrow 0, \quad \text { and } \mathcal{O V}(\mathbb{C}) \cong \mathcal{O} \mathcal{V}_{+}(\mathbb{C}), \quad \text { if } a_{n} \nearrow \infty,
$$

which is easily seen. Thus one may choose the most suitable sequence $\left(a_{n}\right)_{n \in \mathbb{N}}$ for one's purpose without changing the space.

Let us turn to the third part. The following quite technical conditions guarantee a kind of weak reducibility of the projective limit $\mathcal{E} \mathcal{V}(\Omega)$ and in combination with $(P N .1)$ the weak reducibility of $\mathcal{O V}(\Omega)$ too.

Condition (WR) Let $\mathcal{V}:=\left(v_{n}\right)_{n \in \mathbb{N}}$ be a directed family of continuous weights on an open set $\Omega \subset \mathbb{R}^{2}$ and $\left(\Omega_{n}\right)_{n \in \mathbb{N}}$ a family of non-empty open sets such that $\Omega_{n} \neq \mathbb{R}^{2}, \Omega_{n} \subset \Omega_{n+1}$ for all $n \in \mathbb{N}, \mathrm{d}_{n, k}:=\mathrm{d}^{|\cdot|}\left(\Omega_{n}, \partial \Omega_{k}\right)>0$ for all $n, k \in \mathbb{N}, k>n$, and $\Omega=\bigcup_{n \in \mathbb{N}} \Omega_{n}$.

$(W R .1)$ For every $n \in \mathbb{N}$ let there be $g_{n} \in \mathcal{O}(\mathbb{C})$ with $g_{n}(0)=1$ and $\mathbb{N} \ni I_{j}(n)>n$ such that

(a) for every $\varepsilon>0$ there is a compact set $K \subset \bar{\Omega}_{n}$ with $v_{n}(x) \leq \varepsilon v_{I_{1}(n)}(x)$ for all $x \in \Omega_{n} \backslash K$. 
(b) there is an open set $X_{I_{2}(n)} \subset \mathbb{R}^{2} \backslash \bar{\Omega}_{I_{2}(n)}$ such that there are $R_{n}, r_{n} \in \mathbb{R}$ with $0<2 R_{n}<$ $\mathrm{d}^{|\cdot|}\left(X_{I_{2}(n)}, \Omega_{I_{2}(n)}\right):=\mathrm{d}_{X, I_{2}(n)}$ and $R_{n}<r_{n}<\mathrm{d}_{X, I_{2}(n)}-R_{n}$ as well as $A_{2}(\cdot, n): X_{I_{2}(n)}+$ $\mathbb{B}_{R_{n}}(0) \rightarrow(0, \infty), A_{2}(\cdot, n)_{\mid X_{I_{2}(n)}}$ locally bounded, satisfying

$$
\max \left\{\left|g_{n}(\zeta)\right| v_{I_{2}(n)}(z)\left|\zeta \in \mathbb{R}^{2},\right| \zeta-(z-x) \mid=r_{n}\right\} \leq A_{2}(x, n)
$$

for all $z \in \Omega_{I_{2}(n)}$ and $x \in X_{I_{2}(n)}+\mathbb{B}_{R_{n}}(0)$.

(c) for every compact set $K \subset \mathbb{R}^{2}$ there is $A_{3}(n, K)>0$ with

$$
\int_{K} \frac{\left|g_{n}(x-y)\right| v_{n}(x)}{|x-y|} \mathrm{d} y \leq A_{3}(n, K), \quad x \in \Omega_{n} .
$$

( $W R$.2) Let $(W R .1 a)$ be fulfilled. For every $n \in \mathbb{N}$ let there be $\mathbb{N} \ni I_{4}(n)>n$ and $A_{4}(n)>0$ such that

$$
\int_{\Omega_{I_{4}(n)}} \frac{\left|g_{I_{14}(n)}(x-y)\right| v_{p}(x)}{|x-y| v_{k}(y)} \mathrm{d} y \leq A_{4}(n), \quad x \in \Omega_{p},
$$

for $(k, p)=\left(I_{4}(n), n\right)$ and $(k, p)=\left(I_{14}(n), I_{14}(n)\right)$ where $I_{14}(n):=I_{1}\left(I_{4}(n)\right)$.

$(W R .3)$ Let $(W R .1 a),(W R .1 b)$ and $(W R .2)$ be fulfilled. For every $n \in \mathbb{N}$, every closed subset $M \subset \bar{\Omega}_{n}$ and every component $N$ of $M^{C}$ we have

$$
N \cap \bar{\Omega}_{n}^{C} \neq \varnothing \Rightarrow N \cap X_{I_{214}(n)} \neq \varnothing
$$

where $I_{214}(n):=I_{2}\left(I_{14}(n)\right)$, fulfilling $I_{214}(n) \geq I_{14}(n+1)$.

( $W R$ ) is [33, 4.2 Condition, p. 10] combined with the assumption $I_{214}(n) \geq I_{14}(n+1)$, $n \in \mathbb{N}$. We will see that $\Omega_{n}:=\{z \in \mathbb{C}|| \operatorname{Im}(z) \mid<n\}$ and $v_{n}(z):=\exp \left(a_{n}|\operatorname{Re}(z)|^{\gamma}\right)$ for some $0<\gamma \leq 1$ and $a_{n} \nearrow 0$ or $a_{n} \nearrow \infty$ fulfil the conditions above with $g_{n}(z):=\exp \left(-z^{2}\right)$.

Theorem 12 [33, 4.3 Theorem, p. 10] Let $n \in \mathbb{N}$. Then $\pi_{I_{214}(n), n}\left(\mathcal{E} v_{I_{214}(n), \bar{\partial}}\left(\Omega_{I_{214}(n)}\right)\right)$ is dense in $\pi_{I_{14}(n), n}\left(\mathcal{E} v_{I_{14}(n), \bar{\partial}}\left(\Omega_{I_{14}(n)}\right)\right)$ w.r.t. $\left(|\cdot|_{n, m}\right)_{m \in \mathbb{N}_{0}}$ if $(W R)$ is fulfilled.

As a consequence of this theorem, whose proof does not need the assumption $I_{214}(n) \geq$ $I_{14}(n+1)$, we obtain that the projective limit $\mathcal{O V}(\Omega)$ is weakly reduced, which is a generalisation of [32, 5.6 Corollary, p. 69] and [32, 5.11 Corollary, p. 75].

Corollary $13 \mathcal{O V}(\Omega)$ is weakly reduced if $(W R)$ and $(P N .1)$ are satisfied.

Proof Let $n \in \mathbb{N}$. We show that $\pi_{n}(\mathcal{O V}(\Omega))$ is dense in $\pi_{2 J_{1} I_{14}(n), n}\left(\mathcal{O} v_{2 J_{1} I_{14}(n)}\left(\Omega_{2 J_{1} I_{14}(n)}\right)\right)$ w.r.t. $|\cdot|_{n}$ where $J_{1} I_{14}(n):=J_{1}\left(I_{14}(n)\right)$ and

$$
\pi_{n}: \mathcal{O V}(\Omega) \rightarrow \mathcal{O} v_{n}\left(\Omega_{n}\right), \pi_{n}(f):=f_{\mid \Omega_{n}} .
$$

We omit the restriction maps in our proof. Due to Proposition 7 (a) the restrictions to $\Omega_{I_{14}(n)}$ of functions from $\mathcal{O} v_{2 J_{1} I_{14}(n)}\left(\Omega_{2 J_{1} I_{14}(n)}\right)$ are elements of $\mathcal{E} v_{I_{14}(n), \bar{\partial}}\left(\Omega_{I_{14}(n)}\right)$. Let $\varepsilon>0$ and $f_{0} \in \mathcal{O} v_{2 J_{1} I_{14}(n)}\left(\Omega_{2 J_{1} I_{14}(n)}\right)$. For every $j \in \mathbb{N}$ there exists

(i) $f_{j} \in \mathcal{E} v_{I_{214}(n+j-1), \bar{\partial}}\left(\Omega_{I_{214}(n+j-1)}\right)$ with

(ii) $f_{j \mid \Omega_{I_{14}(n+j)}} \in \mathcal{E} v_{I_{14}(n+j), \bar{\partial}}\left(\Omega_{I_{14}(n+j)}\right) \subset \mathcal{O} v_{I_{14}(n+j)}\left(\Omega_{I_{14}(n+j)}\right)$

such that

$$
\left|f_{j}-f_{j-1}\right|_{n+j-1}=\left|f_{j}-f_{j-1}\right|_{n+j-1,0}<\frac{\varepsilon}{2^{j+1}}
$$


by Theorem 12 and the condition $I_{214}(k) \geq I_{14}(k+1)$ for all $k \in \mathbb{N}$ from $(W R)$. Therefore we obtain for every $k \in \mathbb{N}$

$$
\begin{aligned}
\left|f_{k}-f_{0}\right|_{n} & =\left|\sum_{j=1}^{k} f_{j}-f_{j-1}\right|_{n} \leq \sum_{j=1}^{k}\left|f_{j}-f_{j-1}\right|_{n} \leq \sum_{j=1}^{k}\left|f_{j}-f_{j-1}\right|_{n+j-1} \\
& \leq \sum_{(22)}^{k} \frac{\varepsilon}{2^{j+1}}=\frac{\varepsilon}{2}\left(1-\frac{1}{2^{k}}\right)<\frac{\varepsilon}{2} .
\end{aligned}
$$

Now, let $\varepsilon_{0}>0$ and $l \in \mathbb{N}$. We choose $l_{0} \in \mathbb{N}, l_{0} \geq l$, such that $\frac{\varepsilon}{2^{l_{0}+1}}<\varepsilon_{0}$. Similarly, we get for all $p \geq k \geq l_{0}$

$$
\begin{aligned}
&\left|f_{p}-f_{k}\right|_{l} \leq\left|f_{p}-f_{k}\right|_{l_{0}}=\left|\sum_{j=k+1}^{p} f_{j}-f_{j-1}\right|_{l_{0}} \leq \sum_{j=k+1}^{p}\left|f_{j}-f_{j-1}\right|_{l_{0}} \\
& \underset{\substack{l_{0} \leq k \leq j-1 \\
<n+j-1}}{\leq} \sum_{j=k+1}^{p}\left|f_{j}-f_{j-1}\right|_{n+j-1} \leq \sum_{(22)}^{\leq} \sum_{j=k+1}^{p} \frac{\varepsilon}{2^{j+1}}=\frac{\varepsilon}{2}\left(\frac{1}{2^{k}}-\frac{1}{2^{p}}\right)<\frac{\varepsilon}{2^{k+1}} \leq \frac{\varepsilon}{2^{l_{0}+1}}<\varepsilon_{0} .
\end{aligned}
$$

Hence $\left(f_{k}\right)_{k \geq n_{0}}$ is a Cauchy sequence in the Banach space $\mathcal{O} v_{I_{14}\left(n+n_{0}\right)}\left(\Omega_{I_{14}\left(n+n_{0}\right)}\right)$ for every $n_{0} \in \mathbb{N}_{0}$ and thus has a limit $F_{n_{0}} \in \mathcal{O} v_{I_{14}\left(n+n_{0}\right)}\left(\Omega_{I_{14}\left(n+n_{0}\right)}\right)$. These limits coincide on their common domain because for every $n_{1}, n_{2} \in \mathbb{N}_{0}$ with $I_{14}\left(n+n_{1}\right)<I_{14}\left(n+n_{2}\right)$ and $\varepsilon_{1}>0$ there exists $N \in \mathbb{N}$ such that for all $k \geq N$

$$
\begin{aligned}
\left|F_{n_{1}}-F_{n_{2}}\right|_{I_{14}\left(n+n_{1}\right)} & \leq\left|F_{n_{1}}-f_{k}\right|_{I_{14}\left(n+n_{1}\right)}+\left|f_{k}-F_{n_{2}}\right|_{I_{14}\left(n+n_{1}\right)} \\
& \leq\left|F_{n_{1}}-f_{k}\right|_{I_{14}\left(n+n_{1}\right)}+\left|f_{k}-F_{n_{2}}\right|_{I_{14}\left(n+n_{2}\right)}<\frac{\varepsilon_{1}}{2}+\frac{\varepsilon_{1}}{2}=\varepsilon_{1} .
\end{aligned}
$$

We deduce that the glued limit function $f$ given by $f:=F_{n_{0}}$ on $\Omega_{I_{14}\left(n+n_{0}\right)}$ for all $n_{0} \in$ $\mathbb{N}_{0}$ is well-defined and we have $f \in \bigcap_{n_{0} \in \mathbb{N}_{0}} \mathcal{O} v_{I_{14}\left(n+n_{0}\right)}\left(\Omega_{I_{14}\left(n+n_{0}\right)}\right)=\mathcal{O V}(\Omega)$ since $I_{14}\left(n+n_{0}\right) \geq n+n_{0}$. By the definition of $f$ there exists $N \in \mathbb{N}$ such that for every $k \geq N$

$$
\left|f-f_{0}\right|_{n} \leq\left|f-f_{k}\right|_{n}+\left|f_{k}-f_{0}\right|_{n} \underset{n \leq I_{14}(n+0)}{<} \frac{\varepsilon}{2}+\left|f_{k}-f_{0}\right|_{n} \underset{(23)}{\leq} \frac{\varepsilon}{2}+\frac{\varepsilon}{2}=\varepsilon,
$$

which proves our statement.

Combining Theorem 10 and Corollary 13, we obtain the following corollary.

Corollary 14 Let $a_{n} \nearrow 0$ or $a_{n} \nearrow \infty, \mathcal{V}:=\left(\exp \left(a_{n} \mu\right)\right)_{n \in \mathbb{N}}$ and $\Omega_{n}:=S_{n}$ for all $n \in \mathbb{N}$ where

$$
\mu: \mathbb{C} \rightarrow[0, \infty), \mu(z):=|\operatorname{Re}(z)|^{\gamma},
$$

for some $0<\gamma \leq 1$. Then $\mathcal{O V}(\mathbb{C})$ satisfies $(\Omega)$.

Proof We only need to check that the conditions of Theorem 10 are fulfilled. Obviously, $\mu(z)=\mu(|\operatorname{Re}(z)|)$ for all $z \in \mathbb{C}, \mu$ is strictly increasing on $[0, \infty)$ and $\lim _{x \rightarrow \infty, x \in \mathbb{R}} \frac{\ln (1+|x|)}{\mu(x)}=0$. The observation

$$
\mu(x+n)-\mu(x)=|x+n|^{\gamma}-|x|^{\gamma} \leq|x+n-n|^{\gamma}=n^{\gamma}, \quad n \in \mathbb{N}, x \in[0, \infty),
$$

implies that $\mu$ is a strong weight generator with any $\Gamma>1$ and $C:=n^{\gamma}$ by Definition 8 . Let us turn to the conditions $(W R)$ and $(P N .1)$ which we need for the weak reducibility of $\mathcal{O V}(\mathbb{C})$ by Corollary 13. Condition $(P N .1)$ is fulfilled by Example 6. If $a_{n}<0$ for all 
$n \in \mathbb{N}$, then $(W R)$ is fulfilled by [33, 4.10 Example a), p. 22] where we used $\tilde{\mu}(z):=|z|^{\gamma}$ instead of $\mu$, which does not make a difference since

$$
|\operatorname{Re}(z)|^{\gamma} \leq|z|^{\gamma} \leq|\operatorname{Re}(z)|^{\gamma}+n^{\gamma}, \quad z \in \Omega_{n}=S_{n} .
$$

If $a_{n} \geq 0$ for all $n \in \mathbb{N}$, we only have to modify [33, 4.10 Example a), p. 22] a bit. We choose $I_{j}(n):=2 n$ for $j \in\{1,2,4\}$ and define the open set $X_{I_{2}(n)}:=\bar{S}_{4 n}^{C}$. Then we have

$$
I_{214}(n)=8 n \geq 4 n+4=I_{14}(n+1), \quad n \in \mathbb{N} .
$$

Furthermore, we have $\mathrm{d}_{n, k}=|n-k|$ for all $n, k \in \mathbb{N}$.

(WR.1a) and (WR.3): Verbatim as in [33, 4.10 Example a), p. 22].

$(W R .1 b)$ : We have $\mathrm{d}_{X, I_{2}}=2 n$. We choose $g_{n}: \mathbb{C} \rightarrow \mathbb{C}, g_{n}(z):=\exp \left(-z^{2}\right)$, as well as $r_{n}:=1 /(4 n)$ and $R_{n}:=1 /(6 n)$ for $n \in \mathbb{N}$. Let $z=z_{1}+i z_{2} \in \Omega_{I_{2}(n)}=S_{2 n}$ and $x \in X_{I_{2}(n)}+\mathbb{B}_{R_{n}}(0)$. For $\zeta=\zeta_{1}+i \zeta_{2} \in \mathbb{C}$ with $|\zeta-(z-x)|=r_{n}$ we have

$$
\begin{aligned}
\left|g_{n}(\zeta)\right| e^{a_{2 n} \mu(z)} & =e^{-\operatorname{Re}\left(\zeta^{2}\right)} e^{a_{2 n}|\operatorname{Re}(z)|^{\gamma}} \leq e^{-\zeta_{1}^{2}+\zeta_{2}^{2}} e^{a_{2 n}\left(1+\left|z_{1}\right|\right)} \\
& \leq e^{\left(r_{n}+\left|z_{2}\right|+\left|x_{2}\right|\right)^{2}+a_{2 n}\left(1+r_{n}+\left|x_{1}\right|\right)} e^{-\left|\zeta_{1}\right|^{2}+a_{2 n}\left|\zeta_{1}\right|} \\
& \leq e^{\left(r_{n}+2 n+\left|x_{2}\right|\right)^{2}+a_{2 n}\left(1+r_{n}+\left|x_{1}\right|\right)} \sup _{t \in \mathbb{R}} e^{-t^{2}+a_{2 n} t} \\
& =e^{\left(r_{n}+2 n+\left|x_{2}\right|\right)^{2}+a_{2 n}\left(1+r_{n}+\left|x_{1}\right|\right)+a_{2 n}^{2} / 4}=: A_{2}(x, n)
\end{aligned}
$$

and observe that $A_{2}(\cdot, n)$ is continuous and thus locally bounded on $X_{I_{2}(n)}$.

(WR.1c): Let $K \subset \mathbb{C}$ be compact and $x=x_{1}+i x_{2} \in \Omega_{n}$. Then there is $b>0$ such that $|y| \leq b$ for all $y=y_{1}+i y_{2} \in K$ and from polar coordinates and Fubini's theorem it follows that

$$
\begin{aligned}
& \int_{K} \frac{\left|g_{n}(x-y)\right|}{|x-y|} \mathrm{d} y \\
& \leq \underbrace{\sup _{w \in K} e^{a_{2 n}|\operatorname{Re}(w)|}}_{=: C_{1}} \int_{K} \frac{e^{-\operatorname{Re}\left((x-y)^{2}\right)}}{|x-y|} e^{-a_{2 n}\left|y_{1}\right|} \mathrm{d} y \\
& \leq C_{1}\left(\int_{\mathbb{B}_{1}(x)} \frac{e^{-\operatorname{Re}\left((x-y)^{2}\right)}}{|x-y|} e^{-a_{2 n}|\operatorname{Re}(y)|} \mathrm{d} y+\int_{K \backslash \mathbb{B}_{1}(x)} \frac{e^{-\operatorname{Re}\left((x-y)^{2}\right)}}{|x-y|} e^{-a_{2 n}|\operatorname{Re}(y)|} \mathrm{d} y\right) \\
& \leq C_{1}\left(\int_{0}^{2 \pi} \int_{0}^{1} \frac{e^{-r^{2} \cos (2 \varphi)}}{r} e^{-a_{2 n}\left|x_{1}+r \cos (\varphi)\right|} r \mathrm{~d} r \mathrm{~d} \varphi+\int_{K \backslash \mathbb{B}_{1}(x)} e^{-\operatorname{Re}\left((x-y)^{2}\right)} e^{-a_{2 n}|\operatorname{Re}(y)|} \mathrm{d} y\right) \\
& \leq C_{1}\left(2 \pi e^{1+a_{2 n}} e^{-a_{2 n}\left|x_{1}\right|}+\int_{-b}^{b} e^{\left(x_{2}-y_{2}\right)^{2}} \mathrm{~d} y_{2} \int_{\mathbb{R}} e^{-\left(x_{1}-y_{1}\right)^{2}+a_{2 n}\left|x_{1}-y_{1}\right|} \mathrm{d} y_{1} e^{-a_{2 n}\left|x_{1}\right|}\right) \\
& \leq C_{1}\left(2 \pi e^{1+a_{2 n}}+2 b e^{\left(\left|x_{2}\right|+b\right)^{2}} \int_{\mathbb{R}} e^{-y_{1}^{2}+a_{2 n}\left|y_{1}\right|} \mathrm{d} y_{1}\right) e^{-a_{2 n}\left|x_{1}\right|} \\
& =C_{1}\left(2 \pi e^{1+a_{2 n}}+2 b e^{\left(\left|x_{2}\right|+b\right)^{2}} e^{a_{2 n}^{2} / 4} \int_{\mathbb{R}} e^{-\left(\left|y_{1}\right|-a_{2 n} / 2\right)^{2}} \mathrm{~d} y_{1}\right) e^{-a_{2 n}\left|x_{1}\right|} \\
& =C_{1}\left(2 \pi e^{1+a_{2 n}}+4 b e^{\left(\left|x_{2}\right|+b\right)^{2}} e^{a_{2 n}^{2} / 4} \int_{-a_{2 n} / 2}^{\infty} e^{-y_{1}^{2}} \mathrm{~d} y_{1}\right) e^{-a_{2 n}\left|x_{1}\right|} \\
& \leq C_{1}\left(2 \pi e^{1+a_{2 n}}+4 \sqrt{\pi} b e^{(n+b)^{2}+a_{2 n}^{2} / 4}\right) e^{-a_{2 n}\left|x_{1}\right|} .
\end{aligned}
$$


We conclude that $(W R .1 c)$ holds since

$$
e^{-a_{2 n}\left|x_{1}\right|} e^{a_{n}|\operatorname{Re}(x)|^{\gamma}} \leq e^{\left(a_{n}-a_{2 n}\right)\left|x_{1}\right|+a_{n}} \leq e^{a_{n}} .
$$

(WR.2): Let $p, k \in \mathbb{N}$ with $p \leq k$. For all $x=x_{1}+i x_{2} \in \Omega_{p}$ and $y=y_{1}+i y_{2} \in \Omega_{I_{4}(n)}$ we note that

$$
a_{p}|\operatorname{Re}(x)|^{\gamma}-a_{k}|\operatorname{Re}(y)|^{\gamma} \leq a_{k}\left|x_{1}-y_{1}\right|^{\gamma} \leq a_{k}\left(1+\left|x_{1}-y_{1}\right|\right)
$$

because $\left(a_{n}\right)_{n \in \mathbb{N}}$ is non-negative and increasing and $0<\gamma \leq 1$. Like before we deduce that

$$
\begin{aligned}
& \int_{\Omega_{I_{4}(n)}} \frac{\left|g_{n}(x-y)\right| v_{p}(x)}{|x-y| v_{k}(y)} \mathrm{d} y \\
& =\int_{\Omega_{2 n}} \frac{e^{-\operatorname{Re}\left((x-y)^{2}\right)}}{|x-y|} e^{a_{p}|\operatorname{Re}(x)|^{\gamma}-a_{k}|\operatorname{Re}(y)|^{\gamma}} \mathrm{d} y \leq \int_{\Omega_{2 n}} \frac{e^{-\operatorname{Re}\left((x-y)^{2}\right)}}{|x-y|} e^{a_{k}|\operatorname{Re}(x)-\operatorname{Re}(y)|^{\gamma}} \mathrm{d} y \\
& \leq \int_{0}^{2 \pi} \int_{0}^{1} \frac{e^{-r^{2} \cos (2 \varphi)}}{r} e^{a_{k} r^{\gamma}} r \mathrm{~d} r \mathrm{~d} \varphi+\int_{\Omega_{2 n} \backslash \mathbb{B}_{1}(x)} e^{-\operatorname{Re}\left((x-y)^{2}\right)} e^{a_{k}|\operatorname{Re}(x)-\operatorname{Re}(y)|^{\gamma}} \mathrm{d} y \\
& \leq 2 \pi e^{1+a_{k}}+e^{a_{k}} \int_{-2 n}^{2 n} e^{\left(x_{2}-y_{2}\right)^{2}} \mathrm{~d} y_{2} \int_{\mathbb{R}} e^{-\left(x_{1}-y_{1}\right)^{2}+a_{k}\left|x_{1}-y_{1}\right|} \mathrm{d} y_{1} \\
& \leq 2 \pi e^{1+a_{k}}+8 \sqrt{\pi} n e^{a_{k}+\left(\left|x_{2}\right|+2 n\right)^{2}+a_{k}^{2} / 4} \\
& \leq 2 \pi e^{1+a_{I_{14}(n)}}+8 \sqrt{\pi} n e^{a_{I_{14}(n)}+\left(I_{14}(n)+2 n\right)^{2}+a_{I_{14}(n)}^{2} / 4}
\end{aligned}
$$

for $(k, p)=\left(I_{4}(n), n\right)$ and $(k, p)=\left(I_{14}(n), I_{14}(n)\right)$ as $\left(a_{n}\right)_{n \in \mathbb{N}}$ is non-negative and increasing.

We close this section with a special case of our main result on the surjectivity of the Cauchy-Riemann operator on $\mathcal{E} \mathcal{V}(\Omega, E)$. We recall the corresponding result for $E=\mathbb{C}$ which we will need for the application of our main result. It is a consequence of the approximation Theorem 12 in combination with Hörmander's solution of the $\bar{\partial}$-problem in weighted $L^{2}$-spaces [27, Theorem 4.4.2, p. 94] and the Mittag-Leffler procedure.

Theorem 15 [33, 4.8 Theorem, p. 20] Let $(P N)$ with $\psi_{n}(z):=\left(1+|z|^{2}\right)^{-2}, z \in \Omega$, and $(W R)$ be fulfilled and $-\ln v_{n}$ be subharmonic on $\Omega$ for every $n \in \mathbb{N}$. Then

$$
\bar{\partial}: \mathcal{E} \mathcal{V}(\Omega) \rightarrow \mathcal{E} \mathcal{V}(\Omega)
$$

is surjective.

A consequence of this theorem is the following corollary.

Corollary 16 [33, 4.10 Example a), p. 22] Let $\left(a_{n}\right)_{n \in \mathbb{N}}$ be strictly increasing, $a_{n}<0$ for all $n \in \mathbb{N}, \mathcal{V}:=\left(\exp \left(a_{n} \mu\right)\right)_{n \in \mathbb{N}}$ and $\Omega_{n}:=\{z \in \mathbb{C}|| \operatorname{Im}(z) \mid<n\}$ for all $n \in \mathbb{N}$ where

$$
\mu: \mathbb{C} \rightarrow[0, \infty), \mu(z):=|\operatorname{Re}(z)|^{\gamma},
$$

for some $0<\gamma \leq 1$. Then

$$
\bar{\partial}: \mathcal{E V}(\mathbb{C}) \rightarrow \mathcal{E} \mathcal{V}(\mathbb{C})
$$

is surjective. 
The restriction to negative $a_{n}$ comes from the condition that $-\ln v_{n}$ should be subharmonic. We note that the $E$-valued versions of Theorem 15 and Corollary 16 where $E$ is a Fréchet space over $\mathbb{C}$ hold as well by the classical theory of tensor products for nuclear Fréchet spaces (see [33, 4.9 Corollary, p. 21]). Now, we use the results obtained so far to obtain a special case of our main result.

Corollary 17 Let $\mu$ be a subharmonic strong weight generator and $\mathcal{V}:=\left(\exp \left(a_{n} \mu\right)\right)_{n \in \mathbb{N}}$ with $a_{n} \nearrow 0$. Let $(P N)$ with $\psi_{n}(z):=\left(1+|z|^{2}\right)^{-2}, z \in \mathbb{C}$, and $(W R)$ with $\Omega_{n}:=\{z \in$ $\mathbb{C}|| \operatorname{Im}(z) \mid<n\}$ for all $n \in \mathbb{N}$ be fulfilled. If

(a) $E:=F_{b}^{\prime}$ where $F$ is a Fréchet space over $\mathbb{C}$ satisfying $(D N)$, or

(b) E is an ultrabornological PLS-space over $\mathbb{C}$ satisfying (PA),

then

$$
\bar{\partial}^{E}: \mathcal{E} \mathcal{V}(\mathbb{C}, E) \rightarrow \mathcal{E} \mathcal{V}(\mathbb{C}, E)
$$

is surjective.

Proof The space $\mathcal{E} \mathcal{V}(\mathbb{C})$ is nuclear, in particular Schwartz, by [37, Theorem 3.1, p. 188], [37, Remark 2.7, p. 178-179] and [37, Remark 2.3 (b), p. 177] because (PN.1) and (PN.2) ${ }^{1}$ from $(P N)$ are fulfilled. Hence the subspace $\mathcal{E} \mathcal{V}_{\bar{\partial}}(\mathbb{C})=\mathcal{O} \mathcal{V}(\mathbb{C})$ is nuclear by Proposition 7 (b) as well. Further, $\mathcal{O V}(\mathbb{C})$ is weakly reduced by Corollary 13 due to $(W R)$ and thus satisfies $(\Omega)$ by Theorem 10 . Therefore, the assertion is a consequence of the surjectivity of $\bar{\partial}$ in the $\mathbb{C}$-valued case by Theorem 15 and our main result Theorem 5.

Corollary 17 generalises a part of [32, 5.24 Theorem, p. 95] $(K=\varnothing)$ which is the case $\gamma=1$ of the next corollary.

Corollary 18 Let $a_{n} \nearrow 0, \mathcal{V}:=\left(\exp \left(a_{n} \mu\right)\right)_{n \in \mathbb{N}}$ and $\Omega_{n}:=\{z \in \mathbb{C}|| \operatorname{Im}(z) \mid<n\}$ for all $n \in \mathbb{N}$ where

$$
\mu: \mathbb{C} \rightarrow[0, \infty), \mu(z):=|\operatorname{Re}(z)|^{\gamma},
$$

for some $0<\gamma \leq 1$. If

(a) $E:=F_{b}^{\prime}$ where $F$ is a Fréchet space over $\mathbb{C}$ satisfying $(D N)$, or

(b) E is an ultrabornological PLS-space over $\mathbb{C}$ satisfying $(P A)$,

then

$$
\bar{\partial}^{E}: \mathcal{E} \mathcal{V}(\mathbb{C}, E) \rightarrow \mathcal{E} \mathcal{V}(\mathbb{C}, E)
$$

is surjective.

Proof Follows from Corollary 17, (the proof of) Corollary 14 and Example 6.

Acknowledgements Open Access funding provided by Projekt DEAL. The present paper is a generalisation of parts of Chapter 5 of the author's Ph.D Thesis [32], written under the supervision of M. Langenbruch. The author is deeply grateful to him for his support and advice. Further, it is worth to mention that some of the results appearing in the Ph.D Thesis and thus their generalised counterparts in this work are essentially due to him. The author is also grateful to the anonymous reviewer for helpful suggestions improving the present paper. 
Open Access This article is licensed under a Creative Commons Attribution 4.0 International License, which permits use, sharing, adaptation, distribution and reproduction in any medium or format, as long as you give appropriate credit to the original author(s) and the source, provide a link to the Creative Commons licence, and indicate if changes were made. The images or other third party material in this article are included in the article's Creative Commons licence, unless indicated otherwise in a credit line to the material. If material is not included in the article's Creative Commons licence and your intended use is not permitted by statutory regulation or exceeds the permitted use, you will need to obtain permission directly from the copyright holder. To view a copy of this licence, visit http://creativecommons.org/licenses/by/4.0/.

\section{References}

1. Agranovich, M.S.: Partial differential equations with constant coefficients. Rus. Math. Surv. 16(2), 23 (1961). https://doi.org/10.1070/rm1961v016n02abeh004104

2. Bierstedt, K.D., Pietsch, A., Ruess, W.M., Vogt, D. (eds.): Functional Analysis (Proc., Essen, 1991), Lect. Notes in Pure and Appl. Math. 150. Dekker, New York (1994)

3. Bonet, J., Domański, P.: Parameter dependence of solutions of partial differential equations in spaces of real analytic functions. Proc. Am. Math. Soc. 129(2), 495-503 (2001). https://doi.org/10.1090/S00029939-00-05867-6

4. Bonet, J., Domański, P.: Parameter dependence of solutions of differential equations on spaces of distributions and the splitting of short exact sequences. J. Funct. Anal. 230(2), 329-381 (2006). https://doi. org/10.1016/j.jfa.2005.06.007

5. Bonet, J., Domański, P.: The structure of spaces of quasianalytic functions of Roumieu type. Arch. Math. (Basel) 89(5), 430-441 (2007). https://doi.org/10.1007/s00013-007-2073-y

6. Bonet, J., Domański, P.: The splitting of exact sequences of PLS-spaces and smooth dependence of solutions of linear partial differential equations. Adv. Math. 217, 561-585 (2008). https://doi.org/10. 1016/j.aim.2007.07.010

7. Bonet, J., Galbis, A., Meise, R.: On the range of convolution operators on non-quasianalytic ultradifferentiable functions. Studia Math. 126(2), 171-198 (1997). https://doi.org/10.4064/sm-126-2-171-198

8. Braun, R.W.: A partial differential operator which is surjective on Gevrey classes $\Gamma^{d}\left(\mathbb{R}^{3}\right)$ with $1 \leq d<2$ and $d \geq 6$ but not for $2 \leq d<6$. Studia Math. 107(2), 157-169 (1993). https://doi.org/10.4064/sm-1072-157-169

9. Braun, R.W., Meise, R., Taylor, B.A.: Surjectivity of constant coefficient partial differential operators on $\mathcal{A}\left(\mathbb{R}^{4}\right)$ and Whitney's $C_{4}$-cone. Bull. Soc. R. Sci. Liège 70(4-6), 195-206 (2001)

10. Braun, R.W., Meise, R., Vogt, D.: Applications of the projective limit functor to convolution and partial differential equations. In: T. Terziog̃lu (ed.) Advances in the Theory of Fréchet Spaces (Proc., Istanbul, 1988), NATO Sci. Ser. C Math. Phys. Sci., vol. 287, pp. 29-46. Kluwer, Dordrecht (1989). https://doi.org/ 10.1007/978-94-009-2456-7_4

11. Braun, R.W., Meise, R., Vogt, D.: Existence of fundamental solutions and surjectivity of convolution operators on classes of ultra-differentiable functions. Proc. Lond. Math. Soc. (3) 61(2), 344-370 (1990). https://doi.org/10.1112/plms/s3-61.2.344

12. Braun, R.W., Meise, R., Vogt, D.: Characterization of the linear partial differential operators with constant coefficients which are surjective on non-quasianalytic classes of Roumieu type on $\mathbb{R}^{N}$. Math. Nachr. 168(1), 19-54 (1994). https://doi.org/10.1002/mana.19941680103

13. Browder, F.E.: Analyticity and partial differential equations. I. Am. J. Math. 84(4), 666-710 (1962). https://doi.org/10.2307/2372872

14. Cattabriga, L.: Solutions in Gevrey spaces of partial differential equations with constant coefficients. In: Analytic solutions of partial differential equations (Proc., Trento, 1981), Astérisque, vol. 89-90, pp. 129-151. Soc. Math. France, Paris (1981)

15. Dierolf, B.: Splitting theory for PLH spaces. Ph.D. thesis, Universität Trier, Trier (2014). https://doi.org/ 10.25353/ubtr-xxxx-4b2b-53a5

16. Dierolf, B., Sieg, D.: Splitting and parameter dependence in the category of PLH spaces. Rev. R. Acad. Cienc. Exactas Fís. Nat. (Esp.) 113, 59-93 (2019). https://doi.org/10.1007/s13398-017-0424-5

17. Domański, P.: Classical PLS-spaces: spaces of distributions, real analytic functions and their relatives. In: Z. Ciesielski, A. Pełczyński, L. Skrzypczak (eds.) Orlicz Centenary Volume, Banach Center Publications, vol. 64, pp. 51-70. Polish Acad. Sci., Warsaw (2004). https://doi.org/10.4064/bc64-0-5

18. Domański, P.: Real analytic parameter dependence of solutions of differential equations over Roumieu classes. Funct. Approx. Comment. Math. 44(1), 79-109 (2011). https://doi.org/10.7169/facm/ 1301497748 
19. Domański, P., Langenbruch, M.: Vector valued hyperfunctions and boundary values of vector valued harmonic and holomorphic functions. Publ. RIMS Kyoto Univ. 44, 1097-1142 (2008)

20. Epifanov, O.V.: On solvability of the nonhomogeneous Cauchy-Riemann equation in classes of functions that are bounded with weights or systems of weights. Math. Notes 51(1), 54-60 (1992). https://doi.org/ 10.1007/BF01229435

21. Farkas, H., Kawai, T., Kuchment, P., Quinto, T.E., Sternberg, S., Struppa, D., Taylor, B.A.: Remembering leon ehrenpreis (1930-2010). Not. Am. Math. Soc. 58(5), 674-681 (2011)

22. Franken, U., Meise, R.: Generalized Fourier expansions for zero-solutions of surjective convolution operators on $\mathcal{D}^{\prime}(\mathbb{R})$ and $\mathcal{D}_{w}^{\prime}(\mathbb{R})$. Note Mat. X(1), 251-272 (1990). https://doi.org/10.1285/ i15900932v10supn1p251

23. Frerick, L., Kalmes, T.: Some results on surjectivity of augmented semi-elliptic differential operators. Math. Ann. 347(1), 81-94 (2010). https://doi.org/10.1007/s00208-009-0418-5

24. Grothendieck, A.: Produits tensoriels topologiques et espaces nucléaires, 4th edn. Mem. Am. Math. Soc. 16. AMS, Providence (1966). https://doi.org/10.1090/memo/0016

25. Hermanns, V.: Zur Existenz von Rechtsinversen linearer partieller Differentialoperatoren mit konstanten Koeffizienten auf $B_{p, \kappa}^{l o c}(\Omega)$-Räumen. Ph.D. thesis, Universität Wuppertal, Wuppertal (2005)

26. Hörmander, L.: On the existence of real analytic solutions of partial differential equations with constant coefficients. Invent. Math. 21, 151-182 (1973). https://doi.org/10.1007/BF01390194

27. Hörmander, L.: An introduction to complex analysis in several variables, 3rd edn. North-Holland, Amsterdam (1990)

28. Hörmander, L.: The analysis of linear partial differential operators II. Classics Math. Springer, Berlin (2005). https://doi.org/10.1007/b138375

29. Kaballo, W.: Aufbaukurs funktionalanalysis und operatortheorie. Springer, Berlin (2014). https://doi.org/ 10.1007/978-3-642-37794-5

30. Kalmes, T.: Surjectivity of differential operators and linear topological invariants for spaces of zero solutions. Rev. Mat. Complut. 32, 37-55 (2019). https://doi.org/10.1007/s13163-018-0266-5

31. Köthe, G.: Topological vector spaces II Grundlehren Math. Wiss., vol. 237. Springer, Berlin (1979). https://doi.org/10.1007/978-1-4684-9409-9

32. Kruse, K.: Vector-valued fourier hyperfunctions. Ph.D. thesis, Universität Oldenburg, Oldenburg (2014)

33. Kruse, K.: Surjectivity of the $\bar{\partial}$-operator between spaces of weighted smooth vector-valued functions (2018). Arxiv preprint https://arxiv.org/abs/1810.05069v1

34. Kruse, K.: The approximation property for weighted spaces of differentiable functions. In: M. Kosek (ed.) Function Spaces XII (Proc., Kraków, 2018), Banach Center Publ., vol. 119, pp. 233-258. Inst. Math., Polish Acad. Sci., Warszawa (2019). https://doi.org/10.4064/bc119-14

35. Kruse, K.: The inhomogeneous Cauchy-Riemann equation for weighted smooth vector-valued functions on strips with holes (2019). Arxiv preprint https://arxiv.org/abs/1901.02093v2

36. Kruse, K.: Weighted vector-valued functions and the $\varepsilon$-product (2019). Arxiv preprint https://arxiv.org/ abs/1712.01613v6

37. Kruse, K.: On the nuclearity of weighted spaces of smooth functions. Ann. Polon. Math. 124(2), 173-196 (2020). https://doi.org/10.4064/ap190728-17-11

38. Langenbruch, M.: Real roots of polynomials and right inverses for partial differential operators in the space of tempered distributions. Proc. R. Soc. Edinburgh Sect. A 114(3-4), 169-179 (1990). https://doi. org/10.1017/S0308210500024367

39. Langenbruch, M.: Differentiable functions and the $\bar{\partial}$-complex. In: Bierstedt et al. [2], pp. 415-434

40. Langenbruch, M.: Continuous linear right inverses for differential operators. In: D. Przeworska-Rolewicz (ed.) Different Aspects of Differentiability (Proc., Warsaw, 1993), Dissertationes Math., vol. 340, pp. 163-181. Polish Acad. Sci., Warsaw (1995)

41. Langenbruch, M.: Surjective partial differential operators on spaces of ultradifferentiable functions of Roumieu type. Results Math. 29(3), 254-275 (1996). https://doi.org/10.1007/BF0332222300

42. Langenbruch, M.: Surjectivity of partial differential operators on Gevrey classes and extension of regularity. Math. Nachr. 196(1), 103-140 (1998). https://doi.org/10.1002/mana.19981960106

43. Langenbruch, M.: Inheritance of surjectivity for partial differential operators on spaces of real analytic functions. J. Math. Anal. Appl. 297(2), 696-719 (2004). https://doi.org/10.1016/j.jmaa.2004.04.035

44. Langenbruch, M.: Right inverses for partial differential operators on Fourier hyperfunctions. Studia Math. 183(3), 273-299 (2007). https://doi.org/10.4064/sm183-3-5

45. Langenbruch, M.: Right inverses for differential operators on Fourier ultra-hyperfunctions and the property (DN). In: A. Aytuna, R. Meise, T. Terziog̃lu, D. Vogt (eds.) Functional Analysis and Complex Analysis (Proc., Istanbul, 2007), Contemp. Math., vol. 481, pp. 81-104. AMS, Providence (2009). https://doi.org/ $10.1090 /$ conm/481 
46. Langenbruch, M.: Bases in spaces of analytic germs. Ann. Polon. Math. 106, 223-242 (2012). https:// doi.org/10.4064/ap106-0-18

47. Larcher, J.: Surjectivity of differential operators and the division problem in certain function and distribution spaces. J. Math. Anal. Appl. 409(1), 91-99 (2014). https://doi.org/10.1016/j.jmaa.2013.05.078

48. Malgrange, B.: Existence et approximation des solutions des équations aux dérivées partielles et des équations de convolution. Ann. Inst. Fourier (Grenoble) 6, 271-355 (1956). https://doi.org/10.5802/aif. 65

49. Mantlik, F.: Linear equations depending differentiably on a parameter. Integral Equ. Oper. Theory 13(2), 231-250 (1990). https://doi.org/10.1007/BF01193758

50. Mantlik, F.: Partial differential operators depending analytically on a parameter. Ann. Inst. Fourier (Grenoble) 41(3), 577-599 (1991). https://doi.org/10.5802/aif.1266

51. Meise, R., Taylor, B.A., Vogt, D.: Characterization of the linear partial differential operators with constant coefficients that admit a continuous linear right inverse. Ann. Inst. Fourier (Grenoble) 40(3), 619-655 (1990). https://doi.org/10.5802/aif.1226

52. Meise, R., Taylor, B.A., Vogt, D.: Continuous linear right inverses for partial differential operators with constant coefficients and Phragmén-Lindelöf conditions. In: Bierstedt et al. [2], pp. 357-389

53. Meise, R., Taylor, B.A., Vogt, D.: Continuous linear right inverses for partial differential operators on non-quasianalytic classes and on ultradistributions. Math. Nachr. 180(1), 213-242 (1996). https://doi. org/10.1002/mana.3211800110

54. Meise, R., Vogt, D.: Introduction to functional analysis. Oxf. Grad. Texts Math, vol. 2. Clarendon Press, Oxford (1997)

55. Meyer, T.: Surjectivity of convolution operators on spaces of ultradifferentiable functions of Roumieu type. Studia Math. 125(2), 101-129 (1997). https://doi.org/10.4064/sm-125-2-101-129

56. Michael, E.: Continuous selections. I. Ann. Math. (2) 63(2), 361-382 (1956). https://doi.org/10.2307/ 1969615

57. Palamodov, V.P.: Homological methods in the theory of locally convex spaces. Rus. Math. Surv. 26, 1-64 (1971). https://doi.org/10.1070/RM1971v026n01ABEH003815

58. Polyakova, D.A.: Solvability of the inhomogeneous Cauchy-Riemann equation in projective weighted spaces. Sib. Math. J. 58(1), 142-152 (2017). https://doi.org/10.1134/S0037446617010189

59. Schwartz, L.: Théorie des distributions à valeurs vectorielles. I. Ann. Inst. Fourier (Grenoble) 7, 1-142 (1957). https://doi.org/10.5802/aif.68

60. Tönjes, K.: Linear topologische Invarianten für Räume holomorpher Funktionen. Master's thesis, Universität Oldenburg, Oldenburg (2012)

61. Trèves, F.: Fundamental solutions of linear partial differential equations with constant coefficients depending on parameters. Am. J. Math. 84(4), 561-577 (1962). https://doi.org/10.2307/2372862

62. Trèves, F.: Locally convex spaces and linear partial differential equations. Springer, New York (1967). https://doi.org/10.1007/978-3-642-87371-3

63. Vogt, D.: Charakterisierung der Unterräume von s. Math. Z. 155, 109-118 (1977). https://doi.org/10. 1007/BF01214210

64. Vogt, D.: On the solvability of $P(D) f=g$ for vector valued functions. In: H. Komatsu (ed.) Generalized functions and linear differential equations 8 (Proc., Kyoto, 1982), RIMS Kôkyûroku, vol. 508, pp. 168-181. RIMS, Kyoto (1983)

65. Vogt, D.: On the functors $\operatorname{Ext}^{1}(E, F)$ for Fréchet spaces. Studia Math. 85(2), 163-197 (1987). https:// doi.org/10.4064/sm-85-2-163-197

66. Wengenroth, J.: Derived functors in functional analysis. Lecture Notes in Math, vol. 1810. Springer, Berlin (2003). https://doi.org/10.1007/b80165

67. Wengenroth, J.: Surjectivity of partial differential operators with good fundamental solutions. J. Math. Anal. Appl. 379(2), 719-723 (2011). https://doi.org/10.1016/j.jmaa.2011.01.074

Publisher's Note Springer Nature remains neutral with regard to jurisdictional claims in published maps and institutional affiliations. 